\title{
On completely bounded bimodule maps over $\mathrm{W}^{*}$-algebras
}

\author{
by \\ Bojan Magajna (Ljubljana)
}

\begin{abstract}
It is proved that for a von Neumann algebra $A \subseteq \mathrm{B}(\mathcal{H})$ the subspace of normal maps is dense in the space of all completely bounded $A$-bimodule homomorphisms of $\mathrm{B}(\mathcal{H})$ in the point norm topology if and only if the same holds for the corresponding unit balls, which is the case if and only if $A$ is atomic with no central summands of type $I_{\infty, \infty}$. Then a duality result for normal operator modules is presented and applied to the following problem. Given an operator space $X$ and a von Neumann algebra $A$, is the map $q: A \stackrel{e h}{\otimes} X \stackrel{e h}{\otimes} A \rightarrow X \stackrel{n p}{\otimes} A$, induced by $q(a \otimes x \otimes b)=x \otimes a b$, from the extended Haagerup tensor product to the normal version of the Pisier delta tensor product a quotient map? We give a reformulation of this problem in terms of normal extension of some completely bounded maps and answer it affirmatively in the case $A$ is of type I and $X$ belongs to a certain class which includes all finite-dimensional operator spaces.
\end{abstract}

1. Introduction. If $A$ is a von Neumann algebra on a Hilbert space $\mathcal{H}$, it is known (see [7]) that the unit ball $U$ of the space $\operatorname{NCB}_{A}(\mathrm{~B}(\mathcal{H}))_{A}$ of all normal completely bounded (abbreviated CB) $A$-bimodule homomorphisms of $\mathrm{B}(\mathcal{H})$ is dense in the point weak* topology in the unit ball $V$ of the space $\mathrm{CB}_{A}(\mathrm{~B}(\mathcal{H}))_{A}$ of all $\mathrm{CB} A$-bimodule homomorphisms of $\mathrm{B}(\mathcal{H})$. We show in Section 3 that $U$ is dense in $V$ in the point norm (abbreviated p.n.) topology if and only if $A$ is atomic with no central summands of type $I_{\infty, \infty}$. The proof is based on an extension of Arveson's version [1] of Voiculescu's theorem [32] to bimodule mappings over atomic von Neumann algebras and on a recent commutation theorem of Hofmeier and Wittstock [14]. Such a density result implies in particular that, given a finite-dimensional subspace $X \subseteq \mathrm{B}(\mathcal{H})$, each $\phi \in \mathrm{CB}(X, \mathrm{~B}(\mathcal{K}))$ with $\|\phi\|_{\mathrm{cb}}<1$ extends to a normal map $\psi: \mathrm{B}(\mathcal{H}) \rightarrow \mathrm{B}(\mathcal{K})$ with $\|\psi\|_{\mathrm{cb}}<1$.

In Section 4 we observe that every dual operator space $X$ can be embedded into some $\mathrm{B}(\mathcal{H})$ so that each weak* continuous CB map $\phi$ from $X$ into any $\mathrm{B}(\mathcal{K})$ with $\|\phi\|_{\mathrm{cb}}<1$ can be extended to a normal map $\psi$ from $\mathrm{B}(\mathcal{H})$ into $\mathrm{B}(\mathcal{K})$ with $\|\psi\|_{\mathrm{cb}}<1$.

2000 Mathematics Subject Classification: Primary 46L07; Secondary 47L25.

The author is grateful to Gilles Pisier for sending the preprint of [28]. 
An operator $A$-bimodule $X$ over a von Neumann algebra $A \subseteq \mathrm{B}\left(\mathcal{H}_{A}\right)$ is called normal if there exist a Hilbert space $\mathcal{H}$, a complete isometry $\Phi$ : $X \rightarrow \mathrm{B}(\mathcal{H})$ and a (faithful) normal representation $\pi: A \rightarrow \mathrm{B}(\mathcal{H})$ such that $\Phi(a x b)=\pi(a) \Phi(x) \pi(b)$ for all $a, b \in A$ and $x \in X$. If in addition for each $b \in \mathrm{B}(\mathcal{H})$ and each orthogonal family $\left\{e_{i}\right\}_{i \in \mathbb{I}}$ of projections with sum 1 the condition $b \pi\left(e_{i}\right) \in \Phi(X)$ implies that $b \in \Phi(X)$ and similarly for the condition $\pi\left(e_{i}\right) b \in \Phi(X)$, then $X$ is called a strong $A$-bimodule. It follows from [21]-[23] that this definition is independent of the choice of $\Phi$ and $\pi$ and that strong submodules of $\mathrm{B}(\mathcal{H})$ can be characterised as closed in a certain topology and in various other ways. The bimodule dual $X^{\natural}$ of a normal operator $A$-bimodule $X$ is the space $\mathrm{CB}_{A}\left(X, \mathrm{~B}\left(\mathcal{H}_{A}\right)\right)_{A}$ of all completely bounded $A$-bimodule homomorphisms from $X$ to $\mathrm{B}\left(\mathcal{H}_{A}\right)$ (where, for definiteness, we assume that $\mathcal{H}_{A}$ is the Hilbert space on which $A$ is represented in the standard form). Then $X^{\natural}$ is naturally a normal dual operator bimodule (in the sense of [9]) over the commutant $A^{\prime}$ of $A$ in $\mathrm{B}\left(\mathcal{H}_{A}\right)$. Given a normal dual operator bimodule $Y$, we can define its bimodule predual $Y_{\natural}$ as the subspace of its bimodule dual consisting of all weak* continuous mappings. In Section 5 we show that the identity $\left(X^{\natural}\right)_{\natural}=X$ holds for a normal operator $A$-bimodule $X$ if and only if $X$ is strong. (In the special case $A=\mathbb{C}$ this reduces to the well known classical fact.) Then we use this result to study the problem described below.

Pisier [28] proved that for each operator space $X$ and any $\mathrm{C}^{*}$-algebra $A$ the linear map

$$
q_{0}: A \stackrel{h}{\otimes} X \stackrel{h}{\otimes} A \rightarrow X \stackrel{p}{\otimes} A, \quad q_{0}(a \otimes x \otimes b)=x \otimes a b,
$$

is a quotient map, where $A \stackrel{h}{\otimes} X \stackrel{h}{\otimes} A$ is the Haagerup tensor product and $X \stackrel{p}{\otimes} A$ is the completion of $X \otimes A$ in the norm defined by

$$
\left\|\sum_{j=1}^{n} x_{j} \otimes a_{j}\right\|=\sup \left\|\sum_{j=1}^{n} \Phi\left(x_{j}\right) \pi\left(a_{j}\right)\right\|,
$$

where the supremum is over all complete contractions $\Phi: X \rightarrow \mathrm{B}(\mathcal{H})$ and representations $\pi: A \rightarrow \mathrm{B}(\mathcal{H})(\mathcal{H}$ a Hilbert space) such that the range of $\Phi$ is in the commutant of the range of $\pi$.

We shall consider the analogous map for the extended Haagerup tensor product $A \stackrel{e h}{\otimes} X \stackrel{e h}{\otimes} A$, where $A$ is a von Neumann algebra. We shall recall the formal definition of the extended Haagerup tensor product in the next section. Here we just note that the elements of $A \stackrel{e h}{\otimes} X \stackrel{e h}{\otimes} A$ are three-linear completely bounded functionals on $A^{\sharp} \times X^{\sharp} \times A^{\sharp}$ which are weak* continuous in each variable separately and each element of $A \stackrel{e h}{e h} \otimes A$ can be represented 
as a formal sum

$$
\theta=\sum_{i, j \in \mathbb{J}} a_{i} \otimes x_{i j} \otimes b_{j}
$$

where the index set $\mathbb{J}$ is in general infinite and $a_{i} \in A, x_{i j} \in X, b_{j} \in A$ are such that the matrices $a:=\left[a_{i}\right] \in \mathrm{R}_{\mathbb{J}}(A), x:=\left[x_{i j}\right] \in \mathrm{M}_{\mathbb{J}}(X)$ and $b:=\left(b_{j}\right) \in \mathrm{C}_{\mathbb{J}}(A)$ represent bounded operators when $A$ and $X$ are regarded as concrete operator spaces. By definition $\theta$ acts on $(\omega, \tau, \varrho) \in A^{\sharp} \times X^{\sharp} \times A^{\sharp}$ as

$$
\theta(\omega, \tau, \varrho)=\sum_{i, j \in \mathbb{J}} \omega\left(a_{i}\right) \tau\left(x_{i j}\right) \varrho\left(b_{j}\right),
$$

where the sum converges since it represents the product of three bounded operators given respectively by the row matrix $\left[\omega\left(a_{i}\right)\right]$, the $\mathbb{J} \times \mathbb{J}$ matrix $\left[\tau\left(x_{i j}\right)\right]$ and the column matrix $\left(\varrho\left(b_{j}\right)\right)$. The above sum $(1.2)$ will be denoted also by $a \odot x \odot b$. The norm in $A \stackrel{e h}{\otimes} X \stackrel{e h}{\otimes} A$ is given by $\|\theta\|=\inf \|a\|\|x\|\|b\|$, where the infimum is over all possible representations of $\theta$ in the form $\theta=a \odot x \odot b$. By analogy with the space $X \stackrel{p}{\otimes} A$, we can equip $X \otimes A$ with the norm $\|\cdot\|_{n p}$ defined as in (1.1), but considering only normal representations $\pi$ of $A$. However, since the definition of $A \stackrel{e h}{\otimes} X \stackrel{e h}{\otimes} A$ involves infinite sums which are not norm convergent, the norm completion of $X \otimes A$ is in general too small to contain the range of a natural analogue of Pisier's map $q_{0}$. Instead one has to look at the closure $X \stackrel{n p}{\otimes} A$ of $X \otimes A$ in a certain topology, defined more precisely in the next section. Here we just note that by Proposition 5.2, $X \stackrel{n p}{\otimes} A$ can be described as the $A^{\prime}$-bimodule predual of $\mathrm{CB}\left(X, A^{\prime}\right)$, that is,

$$
X \stackrel{n p}{\otimes} A=\mathrm{NCB}_{A^{\prime}}\left(\mathrm{CB}\left(X, A^{\prime}\right), \mathrm{B}\left(\mathcal{H}_{A}\right)\right)_{A^{\prime}}
$$

Using this identification, we may define $q: A \stackrel{e h}{\otimes} X \stackrel{e h}{\otimes} A \rightarrow X \stackrel{n p}{\otimes} A$ by

$$
q(\theta)(\phi)=\sum_{i, j \in \mathbb{J}} a_{i} \phi\left(x_{i j}\right) b_{j} \quad\left(\phi \in \mathrm{CB}\left(X, A^{\prime}\right)\right),
$$

where $\theta$ is represented in the form (1.2). The sum on the right hand side of the above identity represents the product $a\left[\phi\left(x_{i j}\right)\right] b$ of three bounded operators, hence converges, say, in the strong operator topology. We shall indicate in the next section that $q$ is well defined, and then $q$ is clearly a contraction.

Denote by $X^{\sharp}$ the usual operator space dual of $X$ and recall the identification $\mathrm{CB}\left(X, A^{\prime}\right)=X^{\sharp} \stackrel{F}{\otimes} A^{\prime}$ (which may be taken merely as a suggestion that $\mathrm{CB}\left(X, A^{\prime}\right)$ contains a copy of $X^{\sharp} \otimes A^{\prime}$; at this point we do not need the theory of the Fubini tensor product [10]). Observe that each $A^{\prime}$-bimodule homomorphism $\phi: X^{\sharp} \stackrel{F}{\otimes} A^{\prime} \rightarrow \mathrm{B}\left(\mathcal{H}_{A}\right)$ necessarily maps $X^{\sharp} \otimes 1$ into $A^{\prime \prime}=A$, 
hence there is a natural map

$$
\iota: \mathrm{NCB}_{A^{\prime}}\left(X^{\sharp} \stackrel{F}{\otimes} A^{\prime}, \mathrm{B}\left(\mathcal{H}_{A}\right)\right)_{A^{\prime}} \rightarrow \operatorname{NCB}\left(X^{\sharp}, A\right), \quad \iota(\phi)(\varrho)=\phi(\varrho \otimes 1) .
$$

Composing our map $q: A \stackrel{e h}{\otimes} X \stackrel{e h}{\otimes} A \rightarrow X \stackrel{n p}{\otimes} A$ with $\iota$ we get the map studied in [20]. (In $[20,5.1(3)]$ it was already observed that when $X$ is the predual of a von Neumann algebra, the correct target space for $q$ is not $\operatorname{NCB}\left(X^{\sharp}, A\right)$, but a certain subspace of decomposable operators with the decomposable norm.) The following problem is still open in general.

Problem 1. Is the map $q: A \stackrel{e h}{\otimes} X \stackrel{e h}{\otimes} A \rightarrow X \stackrel{n p}{\otimes} A$ a completely quotient map?

It follows from results in [20] that this question has an affirmative answer in the case when $X$ is the predual of a von Neumann algebra. The main obstacle to extending this solution to general operator spaces is that normal completely bounded mappings do not always have normal extensions even if the range space is $\mathrm{B}(\mathcal{H})$.

If we denote by $N$ the kernel of $q$ and put $U=A \stackrel{e h}{\otimes} X \stackrel{e h}{\otimes} A$, the above problem asks if the map $\widetilde{q}: U / N \rightarrow X \stackrel{n p}{\otimes} A$ induced by $q$ is a completely isometric isomorphism. We do not even know if $U / N$ is a normal $A$-bimodule. This is perhaps the main reason that arguments analogous to those used in the proof of Pisier's theorem are not available in the present context of the extended Haagerup tensor product.

We shall show that in case $A \subseteq \mathrm{B}\left(\mathcal{H}_{A}\right)$ is injective the above problem has an affirmative answer for all finite-dimensional operator spaces if and only if there is a conditional expectation $E$ from $\mathrm{B}\left(\mathcal{H}_{A}\right)$ onto $A$ which can be approximated in the point norm topology by a net of normal complete contractions $\phi_{\nu}$ from $\mathrm{B}\left(\mathcal{H}_{A}\right)$ into $A$. We do not require that $\phi_{\nu}$ are $A$ bimodule maps; the results of Section 3 imply that such an approximation of $E$ with $A$-bimodule complete contractions is impossible for a general injective $A$. Even without requiring that $\phi_{\nu}$ are $A$-bimodule maps, such an approximation does not seem very likely for a general injective $A$. The author does not know the answer to the following problem.

Problem 2. For which injective von Neumann algebras $A \subseteq \mathrm{B}(\mathcal{H})$ can a conditional expectation $E: \mathrm{B}(\mathcal{H}) \rightarrow A$ be approximated by normal complete contractions in the point norm topology?

In Section 6 we show that Problem 1 has an affirmative solution in case $A$ is of type I and $X$ satisfies a technical condition (satisfied by all finitedimensional operator spaces); this will also solve Problem 2 for algebras of type I. After a translation of the problem to an extension question for certain normal bimodule mappings (see the diagram in the proof of Corollary 
5.3), the proof is based on the principle of measurable selection if $A$ has a separable predual and then extended to general $A$ of type I. Problem 1 also has an affirmative solution if $X$ is finite-dimensional with 1-exact dual (in the sense of [27]) and $A$ any injective von Neumann algebra (Corollary 6.6).

We refer to [12], [26] and [28] for general theory of operator spaces and completely bounded maps, and to [15] and [19] for some topics that put the problem studied here in a broader perspective.

2. Preliminaries. In this section we shall recall some basic facts about the extended and normal Haagerup tensor products. (For more we refer to [3], [10] and [21]). We shall also show how to extend some recent results of Wittstock and Hofmeier [14] (needed later in this paper) to algebras acting on non-separable spaces.

Given an operator space $Y \subseteq \mathrm{B}(\mathcal{H})$ and an (in general infinite) index set $\mathbb{J}$, we denote by $\mathrm{R}_{\mathbb{J}}(Y), \mathrm{C}_{\mathbb{J}}(Y)$ and $\mathrm{M}_{\mathbb{J}}(Y)$ the spaces of all $1 \times \mathbb{J}, \mathbb{J} \otimes 1$ and $\mathbb{J} \otimes \mathbb{J}$ matrices, respectively, with entries in $Y$ that represent bounded operators. (Equivalently, the supremum of the norms of finite submatrices is finite in each case. Thus $\mathrm{R}_{\mathbb{J}}(Y) \subseteq \mathrm{B}\left(\mathcal{H}^{\mathbb{J}}, \mathcal{H}\right), \mathrm{C}_{\mathbb{J}}(Y) \subseteq \mathrm{B}\left(\mathcal{H}, \mathcal{H}^{\mathbb{J}}\right)$ and $\mathrm{M}_{\mathbb{J}}(Y) \subseteq \mathrm{B}\left(\mathcal{H}^{\mathbb{J}}\right)$.)

The extended Haagerup tensor product of operator spaces

$$
V_{1} \stackrel{e h}{\otimes} \ldots \stackrel{e h}{\otimes} V_{n}
$$

is defined as the space of all $n$-linear completely bounded functionals on

$$
V_{1}^{\sharp} \times \ldots \times V_{n}^{\sharp}
$$

(that is, elements of the dual of $V_{1}^{\sharp} \stackrel{h}{\otimes} \ldots \stackrel{h}{\otimes} V_{n}^{\sharp}$ ) which are weak* continuous in each variable separately. We shall only need the products of at most three spaces. Given three operator spaces $X, Y$ and $Z$, by [10] for each element $\theta \in X \stackrel{e h}{\otimes} Y \stackrel{e h}{\otimes} Z$ there exist an index set $\mathbb{J}$ and matrices $x=\left[x_{i}\right] \in \mathrm{R}_{\mathbb{J}}(X)$, $y=\left[y_{i j}\right] \in \mathrm{M}_{\mathbb{J}}(Y)$ and $z=\left(z_{j}\right) \in \mathrm{C}_{\mathbb{J}}(Z)$ such that

$$
\theta(\xi, \eta, \zeta)=\sum_{i, j \in \mathbb{J}}\left\langle\xi, x_{i}\right\rangle\left\langle\eta, y_{i j}\right\rangle\left\langle\zeta, z_{j}\right\rangle \quad\left(\xi \in X^{\sharp}, \eta \in Y^{\sharp}, \zeta \in Z^{\sharp}\right),
$$

which suggests the notation

$$
\theta=x \odot y \odot z=\sum_{i, j \in \mathbb{J}} x_{i} \otimes y_{i j} \otimes z_{j} .
$$

The norm of $\theta$ is then equal to $\inf \{\|x\|\|y\|\|z\|\}$, where the infimum is over all representations of $\theta$ in the form $\theta=x \odot y \odot z$ such that (2.1) holds.

Following a special case in [7], the normal Haagerup tensor product of dual operator spaces $X^{\sharp}$ and $Y^{\sharp}$ is defined by Effros and Ruan in [10] as the 
dual of $X \stackrel{e h}{\otimes} Y$ :

$$
X^{\sharp} \stackrel{\sigma h}{\otimes} Y^{\sharp}:=(X \stackrel{e h}{\otimes} Y)^{\sharp} .
$$

Products of more than two factors are defined in the same way, but will not be needed here. It is known $([3],[10])$ that $X^{\sharp} \stackrel{h}{\otimes} Y^{\sharp} \subseteq X^{\sharp} \stackrel{e h}{\otimes} Y^{\sharp} \subseteq X^{\sharp} \stackrel{\sigma h}{\otimes} Y^{\sharp}$

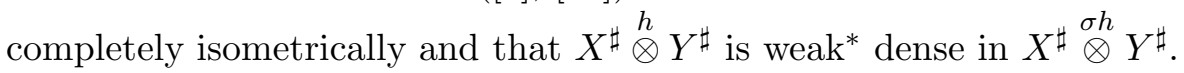

If $A$ is a von Neumann algebra, then $A \stackrel{e h}{\otimes} X \stackrel{e h}{\otimes} A$ can be regarded as the closure of $A \stackrel{h}{\otimes} X \stackrel{h}{\otimes} A$ in a topology the definition of which we now recall.

The $A, B$-topology on a normal operator $A, B$-bimodule $X$, where $A$ and $B$ are von Neumann algebras, is defined by the family of seminorms

$$
s_{\omega}^{\varrho}(x)=\inf \left\{\omega\left(a^{2}\right)^{1 / 2}\|y\| \varrho\left(b^{2}\right)^{1 / 2}: x=a y b, y \in X, a \in A^{+}, b \in B^{+}\right\},
$$

where $\omega$ and $\varrho$ are normal positive functionals on $A$ and $B$, respectively. It is not completely obvious that $s_{\omega}^{\varrho}$ is a seminorm, but we refer to [23] and [22] for more details. Here we only note that if $X$ is a dual normal operator bimodule (in the sense of [9]) this topology is in between the norm and the weak* topology. A bounded linear functional $\theta$ on $X$ is continuous in the $A, B$-topology if and only if for each $x \in X$ the linear functionals $a \mapsto \theta(a x)$ and $b \mapsto \theta(x b)$ are normal on $A$ and $B$ (respectively).

Lemma 2.1. For each operator space $X$ and von Neumann algebra $A$ the Haagerup tensor product $A \stackrel{h}{\otimes} X \stackrel{h}{\otimes} A$ is dense in $A \stackrel{e h}{\otimes} X \stackrel{e h}{\otimes} A$ in the A, A-topology.

Sketch of the proof. Given an element $\theta=\sum_{i, j \in \mathbb{J}} a_{i} \otimes x_{i j} \otimes b_{j}$ as in (2.2), for each finite subset $\mathbb{F} \subseteq \mathbb{J}$ we put $\theta_{\mathbb{F}}=\sum_{i, j \in \mathbb{F}} a_{i} \otimes x_{i j} \otimes b_{j}$. It suffices to prove that the net $\left\{\theta_{\mathbb{F}}\right\}_{\mathbb{F}}$ (where finite subsets of $\mathbb{J}$ are ordered by inclusion) converges to $\theta$ in the $A, A$-topology and this can be done by using a polar decomposition argument in the same way as in the proof of $[22$, Theorem 5.3] or [20, p. 343].

It is easy to see that bounded homomorphisms of normal operator $A, B$ bimodules are continuous in the $A, B$-topology. (For one-sided modules this is proved in [22] and the proof for bimodules is essentially the same.)

Turning to the analogy of Pisier's map $q_{0}$ in the context of the extended Haagerup tensor product, let $S$ be the set of "all" pairs $s=(\Phi, \pi)$ where $\Phi: X \rightarrow \mathrm{B}\left(\mathcal{H}_{s}\right)$ is a complete contraction and $\pi: A \rightarrow \mathrm{B}\left(\mathcal{H}_{s}\right)$ is a normal representation with range contained in $\Phi(X)^{\prime}$. (To assure that $S$ is indeed a set, we may consider only pairs arising by the GNS construction from states on $C^{*}(X) \stackrel{\text { nor }}{\otimes} A$, the normal tensor product defined in [8].) Each such pair 
$s=(\Phi, \pi) \in S$ induces a map $\Phi^{e} \cdot \pi: A \stackrel{e h}{\otimes} X \stackrel{e h}{\otimes} A \rightarrow \mathrm{B}\left(\mathcal{H}_{s}\right)$ by

$$
\left(\Phi^{e} \cdot \pi\right)(\theta)=\sum_{i, j \in \mathbb{J}} \Phi\left(x_{i j}\right) \pi\left(a_{i} b_{j}\right)
$$

where $\theta$ is represented in the form (1.2). Using the above notation $\theta=$ $a \odot x \odot b$, the sum on the right hand side of this definition can be written as $\pi_{1, \mathbb{J}}(a) \Phi_{\mathbb{J}}(x) \pi_{\mathbb{J}, 1}(b)$ (where $\Phi_{\mathbb{J}}, \pi_{\mathbb{J}, 1}$ and $\pi_{1, \mathbb{J}}$ denote the suitable amplifications of $\Phi$ and $\pi$, as usual), which is a product of three bounded operators. (In particular the sum converges in the strong operator topology.) Since the representation of $\theta$ in the form $\theta=a \odot x \odot b$ is not unique, it is not completely obvious that $\Phi^{e} \cdot \pi$ is a well defined map. One way to see this, is to consider first the restriction $\Psi$ of $\Phi^{e} \cdot \pi$ to $A \stackrel{h}{\otimes} X \stackrel{h}{\otimes} A$; then by the well known Wittstock extension theorem for bimodule mappings and using Lemma 2.1 and the automatic continuity of $A$-bimodule homomorphisms in the $A, A$-topology, $\Psi$ has a unique completely bounded $A$-bimodule extension to $A \stackrel{e h}{\otimes} X \stackrel{e h}{\otimes} A$, which must be given by (2.3).

Note that $X \otimes A$ with the norm

$$
\left\|\sum x_{j} \otimes a_{j}\right\|_{n p}=\sup _{(\Phi, \pi) \in S}\left\|\sum_{j} \Phi\left(x_{j}\right) \pi\left(a_{j}\right)\right\|
$$

is an operator $A$-subbimodule of $\mathrm{B}\left(\bigoplus_{s \in S} \mathcal{H}_{s}\right)$ and the direct sum of the mappings $\Phi \stackrel{e}{\odot} \pi$ is a natural contraction $q$ from $A \stackrel{e h}{\otimes} X \stackrel{e h}{\otimes} A$ into $\mathrm{B}\left(\bigoplus_{s \in S} \mathcal{H}_{s}\right)$. We denote the closure of $X \otimes A$ in the $A, A$-topology of $\mathrm{B}\left(\bigoplus_{s \in S} \mathcal{H}_{s}\right)$ by

$$
X \stackrel{n p}{\otimes} A .
$$

Since $q$ is an $A$-bimodule mapping, the range of $q$ is contained in $X \stackrel{n p}{\otimes} A$.

The operator space structure in $X \stackrel{n p}{\otimes} A$ can be described by $\mathrm{M}_{n}(X \stackrel{n p}{\otimes} A)$ $:=X \stackrel{n p}{\otimes} \mathrm{M}_{n}(A)$. Using the identity $\mathrm{M}_{n}(A \stackrel{e h}{\otimes} X \stackrel{e h}{\otimes} A)=\mathrm{C}_{n}(A) \stackrel{e h}{\otimes} X \stackrel{e h}{\otimes} \mathrm{R}_{n}(A)$ $(n \in \mathbb{N})$ it is not difficult to verify that $q$ is a completely contractive $A$ bimodule map.

The following result of Effros and Kishimoto will be needed in Section 3.

TheOREM $2.2([7])$. If $A \subseteq \mathrm{B}(\mathcal{H})$ and $B \subseteq \mathrm{B}(\mathcal{K})$ are von Neumann algebras, then

$$
\mathrm{CB}_{A}(\mathrm{~B}(\mathcal{K}, \mathcal{H}))_{B}=A^{\prime} \stackrel{\sigma h}{\otimes} B^{\prime}
$$

completely isometrically as dual normal operator $A^{\prime}, B^{\prime}$-bimodules. More precisely, the correspondence $\iota\left(a^{\prime} \otimes b^{\prime}\right)(x)=a^{\prime} x b^{\prime}\left(a^{\prime} \in A^{\prime}, b^{\prime} \in B^{\prime}, x \in\right.$ $\mathrm{B}(\mathcal{K}, \mathcal{H}))$ extends to a weak $k^{*}$ continuous completely isometric isomorphism from $A^{\prime} \otimes B^{\prime}$ onto $\mathrm{CB}_{A}\left(\mathrm{~B}(\mathcal{K}, \mathcal{H})_{B}\right.$. Under this isomorphism the subspace 
$A^{\prime} \otimes B^{\prime}$ of $A^{\prime} \otimes B^{\prime}$ is mapped onto the space $\operatorname{NCB}_{A}(\mathrm{~B}(\mathcal{K}, \mathcal{H}))_{B}$ of all normal bimodule maps.

Proof. For $\mathcal{K}=\mathcal{H}$ this is proved in [7]; the general case can be proved in the same way or deduced by standard arguments from the special case by considering $A \oplus B$ acting diagonally on $\mathcal{H} \oplus \mathcal{K}$.

The next theorem, which is a special case of $[9,4.2]$ and can also be deduced by the method of $[14$, p. 149], follows simply by the continuity of module homomorphisms in the $A, \mathbb{C}$-topology.

ThEOREM 2.3. Let $A \subseteq \mathrm{B}(\mathcal{H})$ and $B \subseteq \mathrm{B}(\mathcal{K})$ be von Neumann algebras and $\Phi: \mathrm{B}(\mathcal{K}, \mathcal{H}) \rightarrow \mathrm{B}(\mathcal{K}, \mathcal{H})$ a (not necessarily normal) completely bounded map. If $\Phi$ is a homomorphism of left A-modules, then

$$
\Phi\left(\sum_{j \in \mathbb{J}} a_{j} x_{j}\right)=\sum_{j \in \mathbb{J}} a_{j} \Phi\left(x_{j}\right)
$$

for each index set $\mathbb{J}$ and all $a_{j} \in A, x_{j} \in \mathrm{B}(\mathcal{K}, \mathcal{H})$ such that the sums $\sum_{j \in \mathbb{J}} a_{j} a_{j}^{*}$ and $\sum_{j \in \mathbb{J}} x_{j}^{*} x_{j}$ are weak* convergent. A similar conclusion holds for completely bounded homomorphisms of right $B$-modules. In particular (take $\mathcal{K}=\mathcal{H}$ and $B=A)$, the space $\mathrm{CB}_{A}(\mathrm{~B}(\mathcal{H}))_{A}$ is contained in the commutant of $\mathrm{NCB}_{A^{\prime}}(\mathrm{B}(\mathcal{H}))_{A^{\prime}}$ in $\mathrm{CB}(\mathrm{B}(\mathcal{H}))$ (since each $\Psi \in \mathrm{NCB}_{A^{\prime}}(\mathrm{B}(\mathcal{H}))_{A^{\prime}}$ is of the form $x \mapsto \sum_{j \in \mathbb{J}} a_{j} x b_{j}$ for some index set $\mathbb{J}$ and $a_{j}, b_{j} \in A$ with the sums $\sum_{j \in \mathbb{J}} a_{j} a_{j}^{*}$ and $\sum_{j \in \mathbb{J}} b_{j}^{*} b_{j}$ weak* convergent).

In Section 3 we shall also need the following extract from results of Hofmeier and Wittstock (see $[14,3.1,3.2,3.7]$ ). Recall that an atom in a von Neumann algebra $A$ is a non-zero minimal projection in $A$.

ThEOREM 2.4 ([14]). If $A \subseteq \mathrm{B}(\mathcal{H})$ is a von Neumann algebra such that $A^{\prime}$ contains a von Neumann subalgebra without atoms or, if $A$ has no atomic central direct summands of type $I_{\mathbb{J}, n}$, where $n \in \mathbb{N}$ and $\mathbb{J}$ is an infinite cardinal, then

$$
\left(\mathrm{CB}_{A}(\mathrm{~B}(\mathcal{H}))_{A}\right)^{c}=\mathrm{NCB}_{A^{\prime}}(\mathrm{B}(\mathcal{H}))_{A^{\prime}},
$$

where $\left.\mathrm{CB}_{A}(\mathrm{~B}(\mathcal{H}))_{A}\right)^{c}$ is the commutant of $\mathrm{CB}_{A}(\mathrm{~B}(\mathcal{H}))_{A}$ in $\mathrm{CB}(\mathrm{B}(\mathcal{H}))$.

In [14] Theorem 2.4 was proved for separable spaces only, but the separability assumption was used only to prove certain auxiliary results which imply the following proposition (see $[14,2.5,3.1])$.

Proposition 2.5 ([14]). If $A \subseteq \mathrm{B}(\mathcal{H})$ is an abelian von Neumann algebra without atoms, then

$$
\left(\mathrm{CB}_{A^{\prime}}(\mathrm{B}(\mathcal{H}))_{A^{\prime}}\right)^{c}=\mathrm{NCB}_{A}(\mathrm{~B}(\mathcal{H}))_{A} .
$$

Since Theorem 2.4 can be deduced from Proposition 2.5 as in [14] without assuming the separability of $\mathcal{H}$, we have to prove here Proposition 2.5 only 
for non-separable $\mathcal{H}$. In fact, by Theorem 2.3 only the inclusion

$$
\left(\mathrm{CB}_{A^{\prime}}(\mathrm{B}(\mathcal{H}))_{A^{\prime}}\right)^{c} \subseteq \mathrm{NCB}_{A}(\mathrm{~B}(\mathcal{H}))_{A}
$$

requires additional arguments for non-separable $\mathcal{H}$.

We recall from [16] that a von Neumann algebra $A$ is countably decomposable (also called $\sigma$-finite) if every orthogonal set of non-zero projections in $A$ is countable.

Proof of Proposition 2.5, a reduction to a separable $\mathcal{H}$. Assume first that $A$ is countably decomposable and countably generated. Given a vector $\xi \in$ $\mathcal{H}$, let $p^{\prime} \in A^{\prime}$ be the projection with range $[A \xi]$ and let $p \in A$ be the central carrier of $p^{\prime}$ (the range of $p$ is $\left[A^{\prime} \xi\right]$ ). Then the map $\eta: a p \mapsto a p^{\prime}$ is an isomorphism of $A p$ onto $A p^{\prime}$ [16, p. 335] and (since $\eta^{-1}$ is weak* continuous) it induces a weak ${ }^{*}$ continuous isomorphism $\tilde{\eta}: A p^{\prime} \stackrel{\sigma h}{\otimes} A p^{\prime} \rightarrow A p \stackrel{\sigma h}{\otimes} A p$. By Theorem 2.2 we have weak* homeomorphic completely isometric identifications $\mathrm{CB}_{p^{\prime} A^{\prime} p^{\prime}}\left(p^{\prime} \mathrm{B}(\mathcal{H}) p^{\prime}\right)_{p^{\prime} A^{\prime} p^{\prime}}=A p^{\prime} \stackrel{\sigma h}{\otimes} A p^{\prime}$ and $A p \stackrel{\sigma h}{\otimes} A p=\mathrm{CB}_{A^{\prime} p}(p \mathrm{~B}(\mathcal{H}) p)_{A^{\prime} p}$, hence, composing this with $\widetilde{\eta}$ we get a weak* continuous isomorphism

$$
\tau: \mathrm{CB}_{p^{\prime} A^{\prime} p^{\prime}}\left(p^{\prime} \mathrm{B}(\mathcal{H}) p^{\prime}\right)_{p^{\prime} A^{\prime} p^{\prime}} \rightarrow \mathrm{CB}_{A^{\prime} p}(p \mathrm{~B}(\mathcal{H}) p)_{A^{\prime} p}
$$

For each $\Psi \in \mathrm{CB}_{p^{\prime} A^{\prime} p^{\prime}}\left(p^{\prime} \mathrm{B}(\mathcal{H}) p^{\prime}\right)_{p^{\prime} A^{\prime} p^{\prime}}$ denote $\tau(\Psi)$ by $\widetilde{\Psi}$. If $\Psi$ corresponds to an elementary tensor of the form $a \otimes b \in A p^{\prime} \stackrel{\sigma h}{\otimes} A p^{\prime}$, then one can verify directly that

$$
\widetilde{\Psi} \mid p^{\prime} \mathrm{B}(\mathcal{H}) p^{\prime}=\Psi
$$

By linearity and weak* continuity, (2.5) holds for all

$$
\Psi \in \mathrm{CB}_{p^{\prime} A^{\prime} p^{\prime}}\left(p^{\prime} \mathrm{B}(\mathcal{H}) p^{\prime}\right)_{p^{\prime} A^{\prime} p^{\prime}}
$$

Given $\Phi \in\left(\mathrm{CB}_{A^{\prime}}(\mathrm{B}(\mathcal{H}))_{A^{\prime}}\right)^{c}$, let $\Phi_{p^{\prime}} \in \mathrm{CB}\left(p^{\prime} \mathrm{B}(\mathcal{H}) p^{\prime}\right)$ be defined by $\Phi_{p^{\prime}}(x)=p^{\prime} \Phi(x) p^{\prime}$. Note that $\Phi$ maps $p \mathrm{~B}(\mathcal{H}) p$ into itself since the left and the right multiplication by $p$ are $A^{\prime}$-bimodule mappings, hence commute with $\Phi$. Since each $\widetilde{\Psi} \in \mathrm{CB}_{A^{\prime} p}(p \mathrm{~B}(\mathcal{H}) p)_{A^{\prime} p}$ extends to a map $\widehat{\Psi} \in$ $\mathrm{CB}_{A^{\prime}}(\mathrm{B}(\mathcal{H}))_{A^{\prime}}($ by $\widehat{\Psi}(y)=\widetilde{\Psi}($ pyp $)$ for $y \in \mathrm{B}(\mathcal{H}))$ and $\Phi$ commutes with $\widehat{\Psi}, \Phi \mid p \mathrm{~B}(\mathcal{H}) p$ commutes with each $\widetilde{\Psi}$. Consequently $\Phi_{p^{\prime}}$ commutes with every $\Psi \in \mathrm{CB}_{p^{\prime} A^{\prime} p^{\prime}}\left(p^{\prime} \mathrm{B}(\mathcal{H}) p^{\prime}\right)_{p^{\prime} A^{\prime} p^{\prime}}$. Indeed, since $\widetilde{\Psi}$ is a homomorphism of $A^{\prime} p$-bimodules and $p^{\prime}=p^{\prime} p \in A^{\prime} p$, for each $z \in p^{\prime} \mathrm{B}(\mathcal{H}) p^{\prime} \subseteq p \mathrm{~B}(\mathcal{H}) p$ we have, by (2.5),

$$
\Phi_{p^{\prime}}(\Psi(z))=\Phi_{p^{\prime}}(\widetilde{\Psi}(z))=p^{\prime} \Phi(\widetilde{\Psi}(z)) p^{\prime}=p^{\prime} \widetilde{\Psi}(\Phi(z)) p^{\prime}=\widetilde{\Psi}\left(\Phi_{p^{\prime}}(z)\right) .
$$

Note that $p^{\prime} A^{\prime} p^{\prime}$ is the commutant of $A p^{\prime}$ in $p^{\prime} \mathrm{B}(\mathcal{H}) p^{\prime}$ and $A p^{\prime} \cong A p \subseteq A$ is without atoms. Since the Hilbert space $p^{\prime} \mathcal{H}=[A \xi]$ is separable (for $A$ is countably generated), $\Phi_{p^{\prime}}$ is normal by [14]. Thus for each vector $\xi \in \mathcal{H}$ the 
map $x \mapsto\langle\Phi(x) \xi, \xi\rangle=\left\langle\Phi_{p^{\prime}}(x) \xi, \xi\right\rangle$ is normal on $\mathrm{B}(\mathcal{H})$, which implies that $\Phi$ is normal.

If $A$ is countably decomposable (but not necessarily countably generated), then $A$ contains a countably generated von Neumann subalgebra $B$ without atoms. (To prove this, we may assume that there exists a unit cyclic and separating vector $\xi \in \mathcal{H}$ for $A$ [16, p. 339]. For each $r \in[0,1]$ one can choose a projection $p_{r} \in A$ with $\left\langle p_{r} \xi, \xi\right\rangle=r$. Let $B$ be the weak* closure of the linear span of all the projections $p_{r}$ for (rational) $r$ and let $q^{\prime} \in B^{\prime}$ be the projection onto $[B \xi]$. Since $\left[B^{\prime} \xi\right] \supseteq[A \xi]=\mathcal{H}$, the central carrier of $q^{\prime}$ is 1 , hence $B$ is isomorphic to $B q^{\prime}$. But by the choice of the projections $p_{r}$ the correspondence $\chi_{[0, r]} \mapsto p_{r} \xi$ can be extended to a unitary operator $U$ from $L_{2}[0,1]$ onto $[B \xi]$ such that $U^{*} B q^{\prime} U=L_{\infty}[0,1]$. Since $L_{\infty}[0,1]$ has no atoms the same holds for $B q^{\prime}$ and $B \cong B q^{\prime}$.) Then $A^{\prime} \subseteq B^{\prime}$ implies that

$$
X:=\left(\mathrm{CB}_{A^{\prime}}(\mathrm{B}(\mathcal{H}))_{A^{\prime}}\right)^{c} \subseteq\left(\mathrm{CB}_{B^{\prime}}(\mathrm{B}(\mathcal{H}))_{B^{\prime}}\right)^{c}=: Y .
$$

Since $B$ is countably decomposable and countably generated, $Y$ contains normal maps only, hence so must $X$.

Finally, in general (if $A$ is not countably decomposable), for each projection $p \in A, A p$ has no atoms. The restriction $\Phi_{p}$ of any $\Phi \in\left(\mathrm{CB}_{A^{\prime}}(\mathrm{B}(\mathcal{H}))_{A^{\prime}}\right)^{c}$ to $p \mathrm{~B}(\mathcal{H}) p$ commutes with $\mathrm{CB}_{A^{\prime} p}(p \mathrm{~B}(\mathcal{H}) p)_{A^{\prime} p}$, hence, if $p$ is countably decomposable, $\Phi_{p}$ must be normal. Again, this implies that $\Phi$ is normal since $\langle\Phi(x) \xi, \xi\rangle=\left\langle\Phi_{p}(x) \xi, \xi\right\rangle$ for each vector $\xi \in \mathcal{H}$, where $p$ is the projection with range $\left[A^{\prime} \xi\right]$, which is countably decomposable in $A$.

3. The density in the point norm topology of $\operatorname{NCB}_{A}(\mathrm{~B}(\mathcal{H}))_{A}$ in $\mathrm{CB}_{A}(\mathrm{~B}(\mathcal{H}))_{A}$ for some atomic von Neumann algebras. In this section we shall need an extension of Arveson's version of Voiculescu's theorem [1, Theorem 4] to the case of bimodule maps over certain von Neumann algebras (Theorem 3.2 below). The proof of this theorem relies on the techniques developed in [1] and we shall give below only the necessary adjustments, referring to [1] or [5] for the details. (We have not been able to deduce the result from already known variants of Voiculescu's theorem [17] or [18] since no condition of nuclearity or exactness is present in our situation.) The main result of this section (Theorem 3.5) is stated for atomic von Neumann algebras $A$, but in our application in the later sections we shall only use the case $A=\mathbb{C}$ of the theorem. It follows from Theorem 3.5 that Theorem 3.2 is not true for non-atomic $A$.

A von Neumann algebra is called atomic if it is the weak* closed linear span of its minimal projections, which is the case if and only if $A$ is of type I with atomic centre $[16,6.9 .37]$, hence a direct sum of type I factors.

Lemma 3.1. Let $A$ be a finite atomic von Neumann algebra on a Hilbert space $\mathcal{H}$ such that $A^{\prime}$ is not finite, $\pi: A \rightarrow \mathrm{M}_{n}$ a normal (unital) representa- 
tion (inducing on $\mathrm{M}_{n}$ the structure of an A-bimodule) and $\Phi: \mathrm{B}(\mathcal{H}) \rightarrow \mathrm{M}_{n}$ a unital completely positive (abbreviated CP) A-bimodule homomorphism which annihilates the ideal $\mathrm{K}(\mathcal{H})$ of compact operators. Then for each finite projection $q \in A^{\prime}$ there exists a net of isometries $V_{\nu}: \mathbb{C}^{n} \rightarrow q^{\perp} \mathcal{H}$ such that

$$
V_{\nu} \pi(a)=a V_{\nu} \quad(a \in A) \quad \text { and } \quad \Phi(x)=\lim _{\nu} V_{\nu}^{*} x V_{\nu} \quad(x \in \mathrm{B}(\mathcal{H})),
$$

where the convergence is in the norm topology.

Proof. By the hypothesis we have decompositions of the form

$$
A=\bigoplus_{i \in \mathbb{I}}\left(\mathrm{M}_{n_{i}} \otimes 1_{\mathcal{H}_{i}}\right) \quad \text { and } \quad \mathcal{H}=\bigoplus_{i \in \mathbb{I}}\left(\mathbb{C}^{n_{i}} \otimes \mathcal{H}_{i}\right),
$$

where $n_{i} \in \mathbb{N}$. Since $\pi$ is normal, ker $\pi=p^{\perp} A$ for some central projection $p \in A$. Since $\mathrm{M}_{n}$ is finite-dimensional, $p A=\bigoplus_{i \in \mathbb{F}}\left(\mathrm{M}_{n_{i}} \otimes 1_{\mathcal{H}_{i}}\right)$ for some finite subset $\mathbb{F} \subseteq \mathbb{I}$ and, up to unitary equivalence, $\pi \mid p A$ is of the form

$$
\pi\left(\bigoplus_{i \in \mathbb{F}}\left(a_{i} \otimes 1_{\mathcal{H}_{i}}\right)\right)=\bigoplus_{i \in \mathbb{F}} a_{i}^{\left(k_{i}\right)} \in \bigoplus \mathrm{M}_{n_{i}}^{\left(k_{i}\right)} \subseteq \mathrm{M}_{n}, \quad \sum_{i \in \mathbb{F}} k_{i} n_{i}=n .
$$

Put $\mathcal{H}_{p}=\bigoplus_{i \in \mathbb{F}}\left(\mathbb{C}^{n_{i}} \otimes \mathcal{H}_{i}\right)=p \mathcal{H}$. Since $\Phi$ is an $A$-bimodule homomorphism and $\pi\left(p^{\perp}\right)=0$, we have $\Phi(x)=\Phi(p x p)$ for each $x \in \mathrm{B}(\mathcal{H})$, which means that $\Phi$ may be regarded as a map from $\mathrm{B}\left(\mathcal{H}_{p}\right)$ into $\mathrm{M}_{n}$. By $[1, \mathrm{p} .335]$ there exists a net of contractions $T_{\nu}: \mathbb{C}^{n} \rightarrow \mathcal{H}_{p}$ such that

$$
\Phi(x)=\lim _{\nu} T_{\nu}^{*} x T_{\nu} \quad\left(x \in \mathrm{B}\left(\mathcal{H}_{p}\right)\right) .
$$

Since $p A$ is finite-dimensional there exists a finite group $G$ of unitary elements with linear span $p A$. Let $m$ be the cardinality of $G$. Since $\Phi$ is an $A$-bimodule map, from (3.1) we have

$$
\Phi(x)=\frac{1}{m} \sum_{u \in G} \pi(u) \Phi\left(u^{*} x\right)=\lim _{\nu} \frac{1}{m} \sum_{u \in G} \pi(u) T_{\nu}^{*} u^{*} x T_{\nu}=\lim _{\nu} R_{\nu}^{*} x T_{\nu},
$$

where $R_{\nu}=m^{-1} \sum_{u \in G} u T_{\nu} \pi(u)^{*}$ is a contraction from $\mathbb{C}^{n}$ into $\mathcal{H}_{p}$ satisfying

$$
R_{\nu} \pi(a)=a R_{\nu}
$$

for all $a \in G$, hence for all $a \in p A$. Another averaging over $G$ shows that

$$
\Phi(x)=\lim _{\nu} R_{\nu}^{*} x S_{\nu} \quad(x \in p A),
$$

where $R_{\nu}$ and $S_{\nu}$ are contractions intertwining the representations $\pi \mid p A$ on $\mathbb{C}^{n}$ and $\operatorname{id} \mid p A$ on $\mathcal{H}_{p}$. Since for each unit vector $\xi \in \mathbb{C}^{n}$,

$$
\begin{aligned}
\left\|\left(S_{\nu}-R_{\nu}\right) \xi\right\|^{2} & =\left\|S_{\nu} \xi\right\|^{2}+\left\|R_{\nu} \xi\right\|^{2}-2 \operatorname{Re}\left\langle R_{\nu}^{*} S_{\nu} \xi, \xi\right\rangle \\
& \leq 2\left(1-\operatorname{Re}\left\langle R_{\nu}^{*} S_{\nu} \xi, \xi\right\rangle\right) \stackrel{\nu}{\rightarrow} 0
\end{aligned}
$$

by (3.2), it follows (since $\mathbb{C}^{n}$ is finite-dimensional) that $\lim _{\nu}\left\|S_{\nu}-R_{\nu}\right\|=0$. 
Thus

$$
\Phi(x)=\lim _{\nu} S_{\nu}^{*} x S_{\nu}
$$

for all $x \in \mathrm{B}\left(\mathcal{H}_{p}\right)$; in fact, for all $x \in \mathrm{B}(\mathcal{H})$ since $p^{\perp} S_{\nu}=0$. Now the same arguments as in [1, pp. 336, 337] show that each $S_{\nu}$ can be replaced by an isometry $V_{\nu}$ satisfying all the requirements of the lemma.

TheOREM 3.2. Let $A$ be a finite atomic von Neumann algebra on a Hilbert space $\mathcal{H}$ such that $A^{\prime}$ is not finite, $\pi$ a normal representation of $A$ on a separable Hilbert space $\mathcal{K}, \Phi: \mathrm{B}(\mathcal{H}) \rightarrow \mathrm{B}(\mathcal{K})$ a unital $C P$ A-bimodule homomorphism such that $\Phi(\mathrm{K}(\mathcal{H}))=0$ and $B$ a separable $\mathrm{C}^{*}$-subalgebra of $\mathrm{B}(\mathcal{H})$. Then there exists a sequence of isometries $V_{k}: \mathcal{K} \rightarrow \mathcal{H}$ such that

$$
\begin{aligned}
& V_{k} \pi(a)=a V_{k} \quad(a \in A), \quad \Phi(x)=\lim _{k} V_{k}^{*} x V_{k}, \\
& \Phi(x)-V_{k}^{*} x V_{k} \in \mathrm{K}(\mathcal{K}) \quad \text { for all } x \in B .
\end{aligned}
$$

Sketch of the proof. We have the decompositions

$$
\pi(A)=\bigoplus_{i \in \mathbb{I}}\left(\mathrm{M}_{n_{i}} \otimes 1_{\mathcal{K}_{i}}\right), \quad \mathcal{K}=\bigoplus_{i \in \mathbb{I}}\left(\mathbb{C}^{n_{i}} \otimes \mathcal{K}_{i}\right),
$$

where $n_{i} \in \mathbb{N}$ and $\mathcal{K}_{i}$ are separable Hilbert subspaces of $\mathcal{K}$. Let $C$ be the (separable) $\mathrm{C}^{*}$-subalgebra of $\mathrm{B}(\mathcal{K})$ generated by $\Phi(B) \cup \mathrm{K}(\mathcal{K})$. Using the approximate unit of $\mathrm{K}(\mathcal{K})$ consisting of finite rank projections in $\pi(A)^{\prime}=$ $\bigoplus_{i \in \mathbb{I}}\left(1_{n_{i}} \otimes \mathrm{B}\left(\mathcal{K}_{i}\right)\right)$, by $[1$, pp. 330,331$]$ we may construct a countable quasicentral (for $C$ ) approximate unit $\left(e_{j}\right)$ such that $e_{j} \in \pi(A)^{\prime}$ for all $j \in \mathbb{N}$. Then, given $\varepsilon>0$ and a finite subset $F$ of $C$, the same arguments as in [1, pp. $333,334]$ or $[5$, p. 226] show that there exists a sequence of positive finite rank operators $f_{n} \in \pi(A)^{\prime}$ such that $\sum_{n} f_{n}^{2}=1, x-\sum_{n} f_{n} x f_{n} \in \mathrm{K}(\mathcal{K})$ for $x \in C$ and $\left\|x-\sum_{n} f_{n} x f_{n}\right\|<\varepsilon$ for $x \in F$. Put $E_{n}=f_{1}^{2}+\ldots+f_{n}^{2}$ and let $p_{n} \in \pi(A)^{\prime}$ be the range projection of $E_{n}$. Then $a \mapsto \pi(a) p_{n}$ is a normal representation of $A$ on the finite-dimensional space $p_{n} \mathcal{K}$ and $\Phi_{n}: x \mapsto p_{n} \Phi(x) p_{n}$ is a unital $\mathrm{CP} A$-bimodule homomorphism from $\mathrm{B}(\mathcal{H})$ into $p_{n} \mathrm{~B}(\mathcal{K}) p_{n}=\mathrm{B}\left(p_{n} \mathcal{K}\right)$ such that $\Phi_{n}(\mathrm{~K}(\mathcal{H}))=0$. Now with the use of Lemma 3.1, the required isometries $V_{k}$ can be constructed in the same way as in [1] or [5, pp. 226, 228].

Corollary 3.3. Let $A$ be a finite atomic von Neumann algebra on a Hilbert space $\mathcal{H}$ such that $A^{\prime}$ is not finite, and $\pi$ a normal representation of $A$ on any Hilbert space $\mathcal{K}$. Then any (unital) $C P$ A-bimodule homomorphism $\Phi: \mathrm{B}(\mathcal{H}) \rightarrow \mathrm{B}(\mathcal{K})$ can be approximated in the point norm topology by a net of (unital) normal CP A-bimodule homomorphisms.

Proof. If $\mathcal{K}$ is separable and $\Phi$ is unital with $\Phi(\mathrm{K}(\mathcal{H}))=0$ this follows directly from Theorem 3.2 since each finite subset of $\mathrm{B}(\mathcal{H})$ is contained in a separable $\mathrm{C}^{*}$-subalgebra of $\mathrm{B}(\mathcal{H})$. Let us now remove the assumption that $\mathcal{K}$ is separable. Since $\pi(A)$ is finite and atomic we have a decomposition 
of the form (3.3), which implies that for each $\xi \in \mathcal{K}$ the space $[\pi(A) \xi]$ is separable (namely, $\xi$ has only countably many non-zero components relative to the decomposition of $\mathcal{K}$ in (3.3)). Thus, for each separable subspace $\mathcal{L}$ of $\mathcal{K}$ the space $[\pi(A) \mathcal{L}]$ is also separable. This implies that, given a separable $\mathrm{C}^{*}$-subalgebra $B$ of $\mathrm{B}(\mathcal{H})$ and denoting by $D$ the $\mathrm{C}^{*}$-subalgebra of $\mathrm{B}(\mathcal{K})$ generated by $\Phi(B) \cup \pi(A)$, the space $[D \mathcal{L}]$ is separable. It follows that $\mathcal{K}$ can be decomposed into an orthogonal sum of separable subspaces $\mathcal{K}_{\nu}$ (cyclic for $D$ ) reducing $\Phi(B)$ and $\pi(A)$. Let $p_{\nu} \in \pi(A)^{\prime}$ be the projection with range $\mathcal{K}_{\nu}$ and let $\Phi_{\nu}: \mathrm{B}(\mathcal{H}) \rightarrow \mathrm{B}\left(\mathcal{K}_{\nu}\right)$ be the unital $\mathrm{CP}$ map defined by $\Phi_{\nu}(x)=p_{\nu} \Phi(x) p_{\nu}$. Then $\Phi_{\nu} \mid B$ can be approximated in the p.n. topology by a net of normal unital CP $A$-bimodule maps for each $\nu$, hence the same holds for the orthogonal sum $\Phi\left|B=\sum_{\nu} \Phi_{\nu}\right| B$. Again, since each finite subset of $\mathrm{B}(\mathcal{H})$ is contained in some separable $\mathrm{C}^{*}$-subalgebra of $\mathrm{B}(\mathcal{H}), \Phi$ can be approximated by normal unital CP $A$-bimodule homomorphisms. The same arguments work also if $\Phi$ is not unital (or the restriction that $\Phi$ is unital can be removed by a well known argument, see [12, Lemma 5.1.6]).

For a general CP $A$-bimodule homomorphism $\Phi$ (not necessarily annihilating $\mathrm{K}(\mathcal{H})$ ) let $\Phi=\Phi_{\text {nor }}+\Phi_{\text {sing }}$ be the decomposition into the normal and singular parts [16, Section 10.1], [31]. Note that $\Phi_{\text {nor }}$ and $\Phi_{\text {sing }}$ are CP $A$-bimodule homomorphisms. Since $\Phi_{\text {sing }}(\mathrm{K}(\mathcal{H}))=0$, it follows from the previous paragraph that there exists a bounded net of normal CP $A$-bimodule maps $\Phi_{\nu}: \mathrm{B}(\mathcal{H}) \rightarrow \mathrm{B}(\mathcal{K})$ converging to $\Phi_{\text {sing }}$ in the p.n. topology, hence the net $\left(\Phi_{\nu}+\Phi_{\text {nor }}\right)_{\nu}$ approximates $\Phi$ in the required way. (If $\Phi$ is unital, then a standard modification produces a required net of normal unital maps approximating $\Phi$.)

The following is a variation on [14, Lemma 3.5].

Lemma 3.4. Let $A \subseteq \mathrm{B}(\mathcal{H})$ and $B \subseteq \mathrm{B}(\mathcal{K})$ be von Neumann algebras. If $A$ or $B$ is atomic with finite commutant then

$$
\mathrm{CB}_{A}(\mathrm{~B}(\mathcal{K}, \mathcal{H}))_{B}=\mathrm{NCB}_{A}(\mathrm{~B}(\mathcal{K}, \mathcal{H}))_{B}
$$

Proof. Suppose that $A$ is atomic with $A^{\prime}$ finite (the case when $B$ is atomic and $B^{\prime}$ finite can be treated in the same way or by taking adjoints). Thus we have $A=\bigoplus_{i \in \mathbb{I}}\left(\mathrm{B}\left(\mathcal{H}_{i}\right) \otimes 1_{n_{i}}\right)$ and $\mathcal{H}=\bigoplus_{i \in \mathbb{I}} \mathcal{H}_{i}^{n_{i}}$ for some index set $\mathbb{I}$ and integers $n_{i} \in \mathbb{N}$. Let $p_{i} \in A$ be the projection with range $\mathcal{H}_{i}^{n_{i}}$. Each $\Phi \in \mathrm{CB}_{A}(\mathrm{~B}(\mathcal{K}, \mathcal{H}))_{B}$ determines a collection of maps

$$
\Phi_{i}:=\left.\Phi\right|_{\mathrm{B}\left(\mathcal{K}, \mathcal{H}_{i}^{n_{i}}\right)}=\left.p_{i} \Phi\right|_{\mathrm{B}\left(\mathcal{K}, \mathcal{H}_{i}^{n_{i}}\right)} \in \mathrm{CB}_{\mathrm{B}\left(\mathcal{H}_{i}\right)}\left(\mathrm{B}\left(\mathcal{K}, \mathcal{H}_{i}^{n_{i}}\right)\right)_{B} \quad(i \in \mathbb{I}) .
$$

Since $\Phi$ is a homomorphism of left $A$-modules, for each $x \in \mathrm{B}(\mathcal{K}, \mathcal{H})$ we have, by Theorem 2.3 ,

$$
\Phi(x)=\sum_{i \in \mathbb{I}} p_{i} \Phi\left(p_{i} x\right)=\sum_{i \in \mathbb{I}} p_{i} \Phi_{i}\left(p_{i} x\right) \quad(x \in \mathrm{B}(\mathcal{K}, \mathcal{H})) .
$$


Since the commutant of $\mathrm{B}\left(\mathcal{H}_{i}\right)$ in $\mathrm{B}\left(\mathcal{H}_{i}^{n_{i}}\right)$ is $\mathrm{M}_{n_{i}}$ (and noting that $\mathrm{M}_{n} \stackrel{\sigma h}{\otimes} Y=$ $\mathrm{M}_{n} \stackrel{e h}{\otimes} Y=\mathrm{M}_{n} \stackrel{h}{\otimes} Y$ for each dual operator space $Y$ and $n \in \mathbb{N}$ ), Theorem 2.2 shows that

$$
\mathrm{CB}_{\mathrm{B}\left(\mathcal{H}_{i}\right)}\left(\mathrm{B}\left(\mathcal{K}, \mathcal{H}_{i}^{n_{i}}\right)\right)_{B}=\mathrm{M}_{n_{i}} \stackrel{\sigma h}{\otimes} B^{\prime}=\mathrm{M}_{n_{i}} \stackrel{h}{\otimes} B^{\prime}=\operatorname{NCB}_{\mathrm{B}\left(\mathcal{H}_{i}\right)}\left(\mathrm{B}\left(\mathcal{K}, \mathcal{H}^{n_{i}}\right)\right)_{B},
$$

hence each $\Phi_{i}$ in (3.4) is normal. That $\Phi$ is normal then follows from (3.4) by applying to both sides any vector functional $\omega_{\xi, \eta}$ on $\mathrm{B}(\mathcal{K}, \mathcal{H})$ and using the Schwarz inequality and orthogonality of the family $\left(p_{i}\right)$ to show that the sum for $\omega_{\xi, \eta} \Phi$ is norm convergent.

TheOREM 3.5. Let $A$ be a von Neumann algebra on a Hilbert space $\mathcal{H}$. The space $\mathrm{NCB}_{A}(\mathrm{~B}(\mathcal{H}))_{A}$ is dense in $\mathrm{CB}_{A}(\mathrm{~B}(\mathcal{H}))_{A}$ in the point norm topology if and only if $A$ is atomic without central summands of type $I_{\mathbb{I}, \mathbb{J}}$ where both $\mathbb{I}$ and $\mathbb{J}$ are infinite cardinals; in this case the unit ball of $\operatorname{NCB}_{A}(\mathrm{~B}(\mathcal{H}))_{A}$ is dense in the unit ball of $\mathrm{CB}_{A}(\mathrm{~B}(\mathcal{H}))_{A}$ in the p.n. topology.

Proof. Let $A=A_{a} \oplus B$ be the central decomposition of $A$ into its atomic part $A_{a}$ and the part $B$ without atoms and let $\mathcal{H}=\mathcal{H}_{a} \oplus \mathcal{K}$ be the corresponding decomposition of $\mathcal{H}$. As mentioned in [14] (without proof), if $B \neq 0$, then there exists a non-zero $\Phi \in \operatorname{SCB}_{B}(\mathrm{~B}(\mathcal{K}))_{B}(=$ the space of all singular $B$-bimodule homomorphisms on $\mathrm{B}(\mathcal{K}))$. To show this, note that the restriction to $B$ of the quotient homomorphism $\sigma: \mathrm{B}(\mathcal{K}) \rightarrow$ $\mathrm{B}(\mathcal{K}) / \mathrm{K}(\mathcal{K})$ is completely isometric since $B$ has no atoms, and by the Wittstock extension theorem the map $(\sigma \mid B)^{-1}$ can be extended to a map $\widetilde{\Phi} \in$ $\mathrm{CB}_{B}(\mathrm{~B}(\mathcal{K}) / \mathrm{K}(\mathcal{K}), \mathrm{B}(\mathcal{K}))_{B}$; put $\Phi=\widetilde{\Phi} \sigma$. By Theorem 2.4,

$$
\left(\mathrm{CB}_{B^{\prime}}(\mathrm{B}(\mathcal{K}))_{B^{\prime}}\right)^{c}=\mathrm{NCB}_{B}(\mathrm{~B}(\mathcal{K}))_{B}
$$

hence

$$
\Phi \notin\left(\mathrm{CB}_{B^{\prime}}(\mathrm{B}(\mathcal{K}))_{B^{\prime}}\right)^{c} .
$$

On the other hand, $\left(\mathrm{CB}_{B^{\prime}}(\mathrm{B}(\mathcal{K}))_{B^{\prime}}\right)^{c}$ contains $\mathrm{NCB}_{B}(\mathrm{~B}(\mathcal{K}))_{B}$ by Theorem 2.3 , hence it also contains the p.n. closure of $\operatorname{NCB}_{B}(\mathrm{~B}(\mathcal{K}))_{B}$. It follows that

$$
\Phi \notin{\overline{\mathrm{NCB}_{B}(\mathrm{~B}(\mathcal{K}))_{B}}}^{\mathrm{p} . \mathrm{n} .} \text {. }
$$

Thus $\overline{\operatorname{NCB}_{B}(\mathrm{~B}(\mathcal{K}))_{B}}$ p.n. $\neq \mathrm{CB}_{B}(\mathrm{~B}(\mathcal{K}))_{B}$, which easily implies that

$$
\overline{\mathrm{NCB}}_{A}(\mathrm{~B}(\mathcal{H}))_{A} \text { p.n. } \neq \mathrm{CB}_{A}(\mathrm{~B}(\mathcal{H}))_{A} .
$$

A similar argument can be applied if $A$ contains central summands of type $I_{\infty, \infty}$ (since such a summand contains a von Neumann algebra without atoms and we can apply Theorem 2.4 again to this summand). Thus, if $\mathrm{NCB}_{A}(\mathrm{~B}(\mathcal{H}))_{A}$ is p.n. dense in $\mathrm{CB}_{A}(\mathrm{~B}(\mathcal{H}))_{A}$ then $A$ must be atomic with no central summands of type $I_{\infty, \infty}$.

Assume now that $A$ is atomic with a central decomposition $A=A_{1} \oplus A_{2}$, $\mathcal{H}=\mathcal{H}_{1} \oplus \mathcal{H}_{2}$, where $A_{1}$ is finite with $A_{1}^{\prime}$ infinite and $A_{2}^{\prime}$ is finite $\left(A_{1}\right.$ or $A_{2}$ 
may be absent). Then each $\Phi \in \mathrm{CB}_{A}(\mathrm{~B}(\mathcal{H}))_{A}$ has a decomposition $\Phi=\left[\Phi_{i j}\right]$, where $\Phi_{i j} \in \mathrm{CB}_{A_{i}}\left(\mathrm{~B}\left(\mathcal{H}_{j}, \mathcal{H}_{i}\right)\right)_{A_{j}}(i, j=1,2)$. If $\Phi$ is singular and $\mathrm{CP}$ then by Lemma $3.4, \Phi_{i j}=0$ if $(i, j) \neq(1,1)$. By Corollary 3.3, $\Phi_{1,1}$ is in the p.n. closure of the normal CP $A_{1}$-bimodule homomorphisms, hence $\Phi$ is in the p.n. closure of the normal CP $A$-bimodule maps. If $\Phi$ is unital CP (but not necessarily singular), we may decompose $\Phi$ as $\Phi_{\text {nor }}+\Phi_{\text {sing }}$ and approximate $\Phi_{\text {sing }}$ by normal CP $A$-bimodule maps $\Phi_{k}$; then modifying the CP maps $\Phi_{\text {nor }}+\Phi_{k}$ in the standard way to assure unitality, we get an approximation of $\Phi$ in the p.n. topology by a net of unital normal CP $A$-bimodule maps. Finally, the case of a general map $\Phi \in \mathrm{CB}_{A}(\mathrm{~B}(\mathcal{H}))_{A}$ can be reduced to the completely positive case by Paulsen's well known $2 \times 2$ matrix technique (see $[26])$.

4. A proper embedding of a dual operator space. It is well known that each dual operator space $X^{\sharp}$ can be represented completely isometrically and weak* homeomorphically as a weak* closed subspace in some $\mathrm{B}(\mathcal{H})$ $\left[12\right.$, p. 45], but in general, weak* continuous $\mathrm{CB}$ mappings from $X^{\sharp}$ to $\mathrm{B}(\mathcal{K})$ do not extend to weak* continuous $\mathrm{CB}$ mappings from $\mathrm{B}(\mathcal{H})$ into $\mathrm{B}(\mathcal{K})$ (see [9] and [2]). We say that $X^{\sharp} \subseteq \mathrm{B}(\mathcal{H})$ is properly embedded if each weak* continuous map $\phi: X^{\sharp} \rightarrow \mathrm{B}(\mathcal{K})$ with $\|\phi\|_{\text {cb }}<1$ (for each Hilbert space $\mathcal{K}$ ) can be extended to a weak* continuous map $\widetilde{\phi}: \mathrm{B}(\mathcal{H}) \rightarrow \mathrm{B}(\mathcal{K})$ with $\|\widetilde{\phi}\|_{\mathrm{cb}}<1$. (All maps here are linear.)

Proposition 4.1. Each dual operator space $X^{\sharp}$ can be properly embedded into some $\mathrm{B}(\mathcal{H})$. More precisely, there exist a Hilbert space $\mathcal{H}$ and a weak ${ }^{*}$ homeomorphic complete isometry $\Phi$ from $X^{\sharp}$ onto a weak ${ }^{*}$ closed subspace of $\mathrm{B}(\mathcal{H})$ such that for each weak ${ }^{*}$ continuous map $\psi: X^{\sharp} \rightarrow \mathrm{B}(\mathcal{K})$ with $\|\psi\|_{\mathrm{cb}}<1$ (K $\mathcal{K}$ any Hilbert space) there exists a weak ${ }^{*}$ continuous map $\widetilde{\psi}: \mathrm{B}(\mathcal{H}) \rightarrow \mathrm{B}(\mathcal{K})$ satisfying $\widetilde{\psi} \Phi=\psi$ and $\|\widetilde{\psi}\|_{\mathrm{cb}}<1$.

Proof. Let $\mathcal{K}$ be a fixed Hilbert space of dimension equal to the cardinality of some weak* dense subset of $X^{\sharp}$ and let $S$ be the set of all weak* continuous maps $\psi: X^{\sharp} \rightarrow \mathrm{B}(\mathcal{K})$ such that $\|\psi\|_{\mathrm{cb}}<1$. Let

$$
\Phi=\bigoplus_{\psi \in S} \psi, \quad \mathcal{H}=\bigoplus_{\psi \in S} \mathcal{K} .
$$

For each $\psi \in S$ let $\iota_{\psi}: \mathcal{K} \rightarrow \mathcal{H}$ be the corresponding inclusion, $p_{\psi}: \mathcal{H} \rightarrow \mathcal{K}$ the projection and define

$$
\widetilde{\psi}: \mathrm{B}(\mathcal{H}) \rightarrow \mathrm{B}(\mathcal{K}) \quad \text { by } \quad \widetilde{\psi}(v)=p_{\psi} v \iota_{\psi} .
$$

Then $\widetilde{\psi} \Phi=\psi$ and clearly $\widetilde{\psi}$ is a weak ${ }^{*}$ continuous complete contraction.

If $\psi: X^{\sharp} \rightarrow \mathrm{B}(\mathcal{L})$ is weak ${ }^{*}$ continuous with $\|\psi\|_{\mathrm{cb}}<1(\mathcal{L}$ any Hilbert space), then $\mathcal{L}$ can be decomposed as an orthogonal sum of subspaces $\mathcal{K}_{\nu}$ 
reducing $\psi\left(X^{\sharp}\right)$ such that $\operatorname{dim} \mathcal{K}_{\nu} \leq \operatorname{dim} \mathcal{K}$ for each $\nu$; hence we may assume that $\mathcal{K}_{\nu} \subseteq \mathcal{K}$ and construct the required extension $\widetilde{\psi}$ of $\psi$ by applying the previous paragraph to each component of $\psi$.

Clearly $\Phi$ is weak* continuous and, since $X^{\sharp}$ has a weak* continuous completely isometric representation into some $\mathrm{B}(\mathcal{L}), \Phi$ must be completely isometric. As $\Phi$ is weak* continuous and isometric, it is a well known consequence of the Krĕn-Shmul'yan theorem that $\Phi\left(X^{\sharp}\right)$ is weak* closed and $\Phi$ is a weak* homeomorphism onto $\Phi\left(X^{\sharp}\right)$.

Proposition 4.2. Let $X^{\sharp} \subseteq \mathrm{B}(\mathcal{H})$ be a properly embedded dual operator space and let $\eta: \mathrm{T}(\mathcal{H}) \rightarrow X$ be the completely quotient map from the trace class $\mathrm{T}(\mathcal{H})$ to the predual $X$ of $X^{\sharp}$ the adjoint of which is the inclusion of $X^{\sharp}$ into $\mathrm{B}(\mathcal{H})$. Then for all operator spaces $U$ and $V$,

$$
\sigma:=1 \otimes \eta \otimes 1: U \stackrel{e h}{\otimes} \mathrm{T}(\mathcal{H}) \stackrel{e h}{\otimes} V \rightarrow U \stackrel{e h}{\otimes} X \stackrel{e h}{\otimes} V
$$

is a completely quotient map.

Proof. Let $w \in U \stackrel{e h}{\otimes} X \stackrel{e h}{\otimes} V$ and $\|w\|<1$. Then there exist an index set $\mathbb{J}$ and $u \in \mathrm{R}_{\mathbb{J}}(U), x \in \mathrm{M}_{\mathbb{J}}(X)$ and $v \in \mathrm{C}_{\mathbb{J}}(V)$ such that $\|u\|,\|x\|$ and $\|v\|$ are all less than 1 and $w=u \odot x \odot v:=\sum_{i, j \in J} u_{i} \otimes x_{i j} \otimes v_{j}$. Note that the operator space $\mathrm{M}_{\mathbb{J}}(X)$ can be identified naturally with the space $\operatorname{NCB}\left(X^{\sharp}, \mathrm{M}_{\mathbb{J}}\right)$ of all weak* continuous CB mappings from $X^{\sharp}$ into $\mathrm{M}_{\mathbb{J}}$ (by sending each matrix $x=\left[x_{i j}\right]$ to the map $\widehat{x}$ defined by $\widehat{x}(\varrho)=\left[\varrho\left(x_{i j}\right)\right]$ for $\left.\varrho \in X^{\sharp}\right)$. In particular $\mathrm{M}_{\mathbb{J}}(\mathrm{T}(\mathcal{H}))=\operatorname{NCB}\left(\mathrm{B}(\mathcal{H}), \mathrm{M}_{\mathbb{J}}\right)$ since $\mathrm{B}(\mathcal{H})$ is the dual of $\mathrm{T}(\mathcal{H})$. The assumption that $X^{\sharp}$ is properly embedded into $\mathrm{B}(\mathcal{H})$ implies that

$$
\operatorname{NCB}\left(\mathrm{B}(\mathcal{H}), \mathrm{M}_{\mathbb{J}}\right) \rightarrow \operatorname{NCB}\left(X^{\sharp}, \mathrm{M}_{\mathbb{J}}\right), \quad \phi \mapsto \phi \mid X^{\sharp},
$$

is a quotient map, hence the amplification

$$
\eta_{\mathbb{J}}: \mathrm{M}_{\mathbb{J}}(\mathrm{T}(\mathcal{H})) \rightarrow \mathrm{M}_{\mathbb{J}}(X)
$$

of $\eta$ is also a quotient map. So, there exists an element $t \in \mathrm{M}_{\mathbb{J}}(\mathrm{T}(\mathcal{H}))$ such that $\|t\|<1$ and $\eta_{\mathbb{J}}(t)=x$. With $z:=u \odot t \odot v \in U \stackrel{e h}{\otimes} \mathrm{T}(\mathcal{H}) \stackrel{e h}{\otimes} V$, we have now $\|z\|<1$ and $\sigma(z)=w$. This proves that $\sigma$ is a quotient map. That $\sigma$ is in fact a completely quotient map follows by applying the same argument to the spaces $\mathrm{C}_{n}(U)$ and $\mathrm{R}_{n}(V)(n \in \mathbb{N})$ instead of $U$ and $V$, respectively, since

$$
\mathrm{M}_{n}(U \stackrel{e h}{\otimes} X \stackrel{e h}{\otimes} V)=\mathrm{C}_{n}(U) \stackrel{e h}{\otimes} X \stackrel{e h}{\otimes} \mathrm{R}_{n}(V)
$$

isometrically for every operator space $X$.

In the rest of this section $A \subseteq \mathrm{B}(\mathcal{H})$ will be an injective von Neumann algebra and we will say a few words about the approximation of $\mathrm{CB}$ maps from $\mathrm{B}(\mathcal{H})$ into $A$ with normal maps in the p.n. topology. 
Proposition 4.3. If $A$ is an injective von Neumann algebra, then the unit ball $N_{1}$ of $\operatorname{NCB}(\mathrm{B}(\mathcal{H}), A)$ is dense in the unit ball $C_{1}$ of $\mathrm{CB}(\mathrm{B}(\mathcal{H}), A)$ in the p.n. topology if and only if for each finite-dimensional subspace $F$ of $\mathrm{B}(\mathcal{H})$ the restriction $r_{F}: \operatorname{NCB}(\mathrm{B}(\mathcal{H}), A) \rightarrow \mathrm{CB}(F, A), r_{F}(\psi)=\psi \mid F$, is a quotient map.

Proof. Assume that $N_{1}$ is dense in $C_{1}$ in the p.n. topology. Let $F$ be a finite-dimensional subspace of $\mathrm{B}(\mathcal{H}), \phi \in \mathrm{CB}(F, A),\|\phi\|_{\mathrm{cb}}<1$, and choose $\varepsilon$ so that $0<\varepsilon<1-\|\phi\|_{\mathrm{cb}}$. Since $A$ is injective, there exists an extension $\phi_{0} \in \mathrm{CB}(\mathrm{B}(\mathcal{H}), A)$ of $\phi$ such that $\left\|\phi_{0}\right\|_{\mathrm{cb}}=\|\phi\|_{\mathrm{cb}}$. By the hypothesis there exists a map $\psi_{0} \in \operatorname{NCB}(\mathrm{B}(\mathcal{H}), A)$ such that $\left\|\psi_{0}\right\|_{\mathrm{cb}}<1-\varepsilon$ and $\left\|\left(\phi_{0}-\psi_{0}\right) \mid F\right\|_{\mathrm{cb}}<\varepsilon / 2$. By injectivity again, there exists an extension $\phi_{1} \in \mathrm{CB}(\mathrm{B}(\mathcal{H}), A)$ of $\left(\phi_{0}-\psi_{0}\right) \mid F$ such that $\left\|\phi_{1}\right\|_{\mathrm{cb}}<\varepsilon / 2$. Then by the hypothesis again there exists $\psi_{1} \in \operatorname{NCB}(\mathrm{B}(\mathcal{H}), A)$ such that $\left\|\psi_{1}\right\|_{\mathrm{cb}}<\varepsilon / 2$ and $\left\|\left(\phi_{1}-\psi_{1}\right) \mid F\right\|_{\mathrm{cb}}<\varepsilon / 2^{2}$. Continuing in this way, we find two sequences of maps $\phi_{n} \in \mathrm{CB}(\mathrm{B}(\mathcal{H}), A)$ and $\psi_{n} \in \operatorname{NCB}(\mathrm{B}(\mathcal{H}), A)$ such that $\left\|\phi_{n}\right\|_{\mathrm{cb}}<2^{-n} \varepsilon$ and $\left\|\psi_{n}\right\|_{\mathrm{cb}}<2^{-n} \varepsilon$ if $n \geq 1,\left\|\left(\phi_{n}-\psi_{n}\right) \mid F\right\|_{\mathrm{cb}}<2^{-n-1} \varepsilon$ and $\phi_{n}$ is an extension of $\left(\phi_{0}-\psi_{0}-\ldots-\psi_{n-1}\right) \mid F$. Put $\psi=\sum_{n=0}^{\infty} \psi_{n}$. Then $\psi \in \operatorname{NCB}(\mathrm{B}(\mathcal{H}), A)$, $\|\psi\|_{\mathrm{cb}}<1$ and $\psi \mid F=\phi$, hence $r_{F}$ is a quotient map. This proves the proposition in one direction; the reverse direction is obvious.

From Theorem 3.5 (in the special case $A=\mathbb{C}$ ) and Proposition 4.3 we deduce the following consequence.

Corollary 4.4. Each finite-dimensional subspace of $\mathrm{B}(\mathcal{H})$ is properly embedded.

Corollary 4.5. Let $A$ be an injective von Neumann algebra acting on a Hilbert space $\mathcal{H}_{A}$. Then for all Hilbert spaces $\mathcal{H}$ the unit ball of the space $\operatorname{NCB}(\mathrm{B}(\mathcal{H}), A)$ is dense in the unit ball of $\mathrm{CB}(\mathrm{B}(\mathcal{H}), A)$ in the p.n. topology if and only if there exists a conditional expectation $E: \mathrm{B}\left(\mathcal{H}_{A}\right) \rightarrow A$ in the p.n. closure of the unit ball $U$ of $\operatorname{NCB}\left(\mathrm{B}\left(\mathcal{H}_{A}\right), A\right)$.

Proof. Suppose that there is a conditional expectation $E: \mathrm{B}\left(\mathcal{H}_{A}\right) \rightarrow A$ in the p.n. closure of $U$. Let $\phi \in \mathrm{CB}(\mathrm{B}(\mathcal{H}), A)$ with $\|\phi\|_{\mathrm{cb}}<1, \varepsilon>0$ and $F$ a finite-dimensional subspace of $\mathrm{B}(\mathcal{H})$. By Corollary 4.4 there exists a map $\sigma \in \operatorname{NCB}\left(\mathrm{B}(\mathcal{H}), \mathrm{B}\left(\mathcal{H}_{A}\right)\right)$ such that $\|\sigma\|_{\mathrm{cb}}<1$ and $\sigma|F=\phi| F$. By the hypothesis there exists a map $\psi \in \operatorname{NCB}\left(\mathrm{B}\left(\mathcal{H}_{A}\right), A\right)$ such that $\|\psi\|_{\mathrm{cb}} \leq 1$ and $\|(E-\psi) \mid \phi(F)\|_{\mathrm{cb}}<\varepsilon$. Then $\psi \sigma \in \operatorname{NCB}(\mathrm{B}(\mathcal{H}), A),\|\psi \sigma\|_{\mathrm{cb}}<1$ and (since $\phi=E \phi$ and $\sigma|F=\phi| F)\left\|(\phi-\psi \sigma)\left|F\left\|_{\mathrm{cb}}=\right\|(E-\psi) \phi\right| F\right\|_{\mathrm{cb}}<\varepsilon$.

5. The module dual of a normal operator bimodule. The module dual of an operator bimodule over $\mathrm{C}^{*}$-algebras was introduced by $\mathrm{Na}$ [24] and Pop [29]. Here we need such a dual for normal operator bimodules over von Neumann algebras. To simplify the notation we consider only normal 
bimodules over a single von Neumann algebra $A$; the case of bimodules over a pair of von Neumann algebras can be treated in the same way.

For a normal operator $A$-bimodule $X$, the bimodule dual $X^{\natural}$ of $X$ is defined by

$$
X^{\natural}=\mathrm{CB}_{A}\left(X, \mathrm{~B}\left(\mathcal{H}_{A}\right)\right)_{A},
$$

where $\mathcal{H}_{A}$ is the Hilbert space on which $A$ is represented faithfully and normally in some canonical way, say in the standard form [13]. (Here we shall not need any technical properties of the standard form. If $A$ is $\sigma$-finite, we may simply suppose that $A$ on $\mathcal{H}_{A}$ has a cyclic and separating vector, which determines $\left(A, \mathcal{H}_{A}\right)$ up to unitary equivalence.) $X^{\sharp}$ is an $A^{\prime}$-bimodule in the natural way.

If $Y$ is a dual operator $A$-bimodule, then the bimodule predual $Y_{\natural}$ of $Y$ is defined as the subspace of all weak* continuous elements of $Y^{\natural}$. In particular, since $X^{\natural}$ is a weak* closed subspace of $\mathrm{CB}\left(X, \mathrm{~B}\left(\mathcal{H}_{A}\right)\right)=\left(X \hat{\otimes} \mathrm{T}\left(\mathcal{H}_{A}\right)\right)^{\sharp}$, where $\mathrm{T}\left(\mathcal{H}_{A}\right)$ is the trace class of $\mathcal{H}_{A}$, we may consider $\left(X^{\natural}\right)_{\natural}$.

Theorem 5.1. Let $X$ be a normal operator A-bimodule. Then $\left(X^{\natural}\right)_{\natural}$ is the smallest strong A-bimodule containing $X$. In particular, $\left(X^{\natural}\right)_{\natural}=X$ if and only if $X$ is strong.

Proof. Consider first the bimodule $Y=\mathrm{B}\left(\mathcal{H}_{A}^{\mathbb{I}}\right)=\mathrm{M}_{\mathbb{I}}\left(\mathrm{B}\left(\mathcal{H}_{A}\right)\right)$, where $\mathbb{I}$ is some index set and the $A$-bimodule structure on $Y$ is given by the representation $\pi: A \rightarrow \mathrm{B}\left(\mathcal{H}_{A}^{\mathbb{I}}\right), \pi(a)=a^{(\mathbb{I})}$. Then from Theorem 2.2 we deduce (by first considering $\mathrm{CB}_{A}\left(\mathrm{~B}\left(\mathcal{H}_{A}^{\mathbb{I}}\right)\right)_{A}$ and regarding $\mathcal{H}_{A}$ as a subspace in $\mathcal{H}_{A}^{\mathbb{I}}$ ) that

$$
Y^{\natural}=\mathrm{CB}_{A}\left(\mathrm{~B}\left(\mathcal{H}_{A}^{\mathbb{I}}\right), \mathrm{B}\left(\mathcal{H}_{A}\right)\right)_{A}=\mathrm{R}_{\mathbb{I}}\left(A^{\prime}\right) \stackrel{\sigma h}{\otimes} \mathrm{C}_{\mathbb{I}}\left(A^{\prime}\right),
$$

where for each $a^{\prime} \in \mathrm{R}_{\mathbb{I}}\left(A^{\prime}\right)$ and $b^{\prime} \in \mathrm{C}_{\mathbb{I}}\left(A^{\prime}\right)$ the element $a^{\prime} \otimes b^{\prime}$ acts as a map from $\mathrm{B}\left(\mathcal{H}_{A}^{\mathbb{I}}\right)$ to $\mathrm{B}\left(\mathcal{H}_{A}\right)$ by $\left(a^{\prime} \otimes b^{\prime}\right)(x)=a^{\prime} x b^{\prime}\left(x \in \mathrm{B}\left(\mathcal{H}_{A}^{\mathbb{I}}\right)=\mathrm{M}_{\mathbb{I}}\left(\mathrm{B}\left(\mathcal{H}_{A}\right)\right)\right)$. Let $\mathrm{R}_{\mathbb{I}}^{\mathrm{fin}}\left(A^{\prime}\right)$ be the subspace in $\mathrm{R}_{\mathbb{I}}\left(A^{\prime}\right)$ consisting of all rows that have only finitely many non-zero entries and let $\mathrm{C}_{\mathbb{I}}^{\text {fin }}\left(A^{\prime}\right)$ be the analogous space of columns. Since $\mathrm{R}_{\mathbb{I}}^{\mathrm{fin}}\left(A^{\prime}\right) \otimes \mathrm{C}_{\mathbb{I}}^{\mathrm{fin}}\left(A^{\prime}\right)$ is weak* dense in $\mathrm{R}_{\mathbb{I}}\left(A^{\prime}\right) \stackrel{\sigma h}{\otimes} \mathrm{C}_{\mathbb{I}}\left(A^{\prime}\right)$ and each $\Phi \in\left(Y^{\natural}\right)_{\natural}$ is a $A^{\prime}$-bimodule map, $\Phi$ is determined by the values

$$
b_{i j}:=\Phi\left(e_{i}^{T} \otimes e_{j}\right) \in \mathrm{B}\left(\mathcal{H}_{A}\right),
$$

where $e_{j} \in \mathrm{C}_{\mathbb{I}}^{\text {fin }}\left(A^{\prime}\right)(j \in \mathbb{I})$ has 1 in the $j$ th entry and 0 elsewhere. For each subset $\mathbb{F} \subseteq \mathbb{I}$ let $\left(e_{i}^{T}\right)_{i \in \mathbb{F}}$ denote the column and $\left[e_{j}\right]_{j \in \mathbb{F}}$ the row matrix. Note that for a finite $\mathbb{F}$ the matrix

$$
\left[e_{i}^{T} \otimes e_{j}\right]_{i, j \in \mathbb{F}} \in \mathrm{M}_{\mathbb{F}}\left(\mathrm{R}_{\mathbb{I}}\left(A^{\prime}\right) \stackrel{\sigma h}{\otimes} \mathrm{C}_{\mathbb{I}}\left(A^{\prime}\right)\right)=\mathrm{C}_{\mathbb{F}}\left(\mathrm{R}_{\mathbb{I}}\left(A^{\prime}\right)\right) \stackrel{\sigma h}{\otimes} \mathrm{R}_{\mathbb{F}}\left(\mathrm{C}_{\mathbb{I}}\left(A^{\prime}\right)\right)
$$

satisfies

$$
\left\|\left[e_{i}^{T} \otimes e_{j}\right]_{i, j \in \mathbb{F}}\right\|=\left\|\left(e_{i}^{T}\right)_{i \in \mathbb{F}} \odot\left[e_{j}\right]_{j \in \mathbb{F}}\right\| \leq\left\|\left(e_{i}^{T}\right)_{i \in \mathbb{F}}\right\|\left\|\left[e_{j}\right]_{j \in \mathbb{F}}\right\|=1
$$


since $\left[e_{j}\right]_{j \in \mathbb{I}}$ and $\left(e_{i}^{T}\right)_{i \in \mathbb{I}}$ are just the identity matrix. It follows that the matrix $b:=\left[b_{i j}\right]_{i, j \in \mathbb{I}}$ represents a bounded operator with $\|b\| \leq\|\Phi\|_{\mathrm{cb}}$, hence $b \in Y$. From $\Phi\left(e_{i}^{T} \otimes e_{j}\right)=\left(e_{i}^{T} \otimes e_{j}\right)(b)$ for all $i, j \in \mathbb{I}$ (where $e_{i}^{T} \otimes e_{j} \in$ $\left.\mathrm{R}_{\mathbb{I}}\left(A^{\prime}\right) \stackrel{\sigma h}{\otimes} \mathrm{C}_{\mathbb{I}}\left(A^{\prime}\right)=Y^{\natural}\right)$ we conclude that $\Phi$ is the evaluation at $b$. Thus $\left(Y^{\natural}\right)_{\natural}=Y$.

For a general $X$, we may assume that $X \subseteq \mathrm{B}(\mathcal{K})$ for some Hilbert space $\mathcal{K}$, where the $A$-bimodule structure is induced by a normal representation $\pi: A \rightarrow \mathrm{B}(\mathcal{K})$. Moreover, since each normal representation of $A$ is unitarily equivalent to a restriction of a multiple of the identity representation, we may regard $X$ as a subbimodule of the module of the form $Y=\mathrm{B}\left(\mathcal{H}_{A}^{\mathbb{I}}\right)$ considered in the previous paragraph. By the Wittstock extension theorem the inclusion $X \subseteq Y$ induces the (weak* continuous) quotient mapping $q: Y^{\natural} \rightarrow X^{\natural}$. For each $\phi \in\left(X^{\natural}\right)_{\natural}$ the composition $\phi q$ is in $\left(Y^{\natural}\right)_{\natural}$, hence by what we have already proved there exists an element $b \in Y$ such that

$$
\phi(\theta \mid X)=(\phi q)(\theta)=\theta(b)
$$

for all $\theta \in Y^{\natural}$. If $b \notin \bar{X}^{A, A}$, then by [23, Theorem 3.8] we could choose $\theta \in Y^{\natural}$ such that $\theta(X)=0$ and $\theta(b) \neq 0$, but this would contradict (5.1). Thus, $b \in \bar{X}^{A, A}$ and it follows from (5.1) that $\phi(\sigma)=\bar{\sigma}(b)$ for all $\sigma \in X^{\natural}$, where $\bar{\sigma}$ is the (unique) $A, A$-continuous extension of $\sigma$ to $\bar{X}^{A, A}$. Thus, $\left(X^{\natural}\right)_{\natural}=\bar{X}^{A, A}$ as vector spaces. To show that this identification is completely isometric, let $x=\left[x_{i j}\right] \in \mathrm{M}_{n}(X) \subseteq \mathrm{M}_{n}\left(\mathrm{~B}\left(\mathcal{H}_{A}^{\mathbb{I}}\right)\right)$. For each finite subset $\mathbb{F}$ of $\mathbb{I}$ let $p_{\mathbb{F}}^{\prime} \in \mathrm{M}_{\mathbb{I}}\left(A^{\prime}\right)$ be the projection with range $\mathcal{H}_{A}^{\mathbb{F}}$ and observe that $\|x\|$ can be approximated arbitrarily closely by the norms of the matrices $\left[p_{\mathbb{F}}^{\prime} x_{i j} p_{\mathbb{F}}^{\prime}\right]$ as $\mathbb{F}$ ranges over all finite subsets of $\mathbb{I}$. Since each $p_{\mathbb{F}}^{\prime} \mathrm{B}\left(\mathcal{H}_{A}^{\mathbb{I}}\right) p_{\mathbb{F}}^{\prime}=\mathrm{B}\left(\mathcal{H}_{A}^{\mathbb{F}}\right)$ can be identified with $\mathrm{M}_{m}\left(\mathrm{~B}\left(\mathcal{H}_{A}\right)\right)$, where $m$ is the cardinality of $\mathbb{F}$, it follows that $\|x\|=\sup \left\|\left[\varrho_{k l}\left(x_{i j}\right)\right]\right\|$, where the supremum is over all complete contractions $\left[\varrho_{k l}\right] \in \mathrm{M}_{m}\left(X^{\natural}\right)=\mathrm{CB}\left(X, \mathrm{M}_{m}\left(\mathrm{~B}\left(\mathcal{H}_{A}\right)\right)\right)$ and all $m \in \mathbb{N}$.

Given an operator space $X$ and a von Neumann algebra $A \subseteq \mathrm{B}\left(\mathcal{H}_{A}\right)$, we denote the space $\mathrm{CB}\left(X, A^{\prime}\right)$ by $X^{\sharp} \stackrel{F}{\otimes} A^{\prime}$ to emphasise that it contains a copy of $X^{\sharp} \otimes A^{\prime}$. (This is a special case of the general Fubini tensor product [10].)

Proposition 5.2. For each operator space $X$ and von Neumann algebra $A$,

$$
(X \stackrel{n p}{\otimes} A)^{\natural}=X^{\sharp} \stackrel{F}{\otimes} A^{\prime}
$$

completely isometrically as $A^{\prime}$-bimodules, hence

$$
X \stackrel{n p}{\otimes} A=\left(X^{\sharp} \stackrel{F}{\otimes} A^{\prime}\right)_{\natural}=\operatorname{NCB}_{A^{\prime}}\left(X^{\sharp} \stackrel{F}{\otimes} A^{\prime}, \mathrm{B}\left(\mathcal{H}_{A}\right)\right)_{A^{\prime}} .
$$


Proof. By the definitions we have $X^{\sharp} \stackrel{F}{\otimes} A^{\prime}=\mathrm{CB}\left(X, A^{\prime}\right)$ and $(X \stackrel{n p}{\otimes} A)^{\natural}=$ $\mathrm{CB}_{A}\left(X \otimes A, \mathrm{~B}\left(\mathcal{H}_{A}\right)\right)_{A}$. By the definition (2.4) of the norm in $X^{n p} \otimes A$ each complete contraction $\phi \in \mathrm{CB}\left(X, A^{\prime}\right)$ can be extended (uniquely) to a completely contractive $A$-bimodule map $\widetilde{\phi}: X \otimes A \rightarrow \mathrm{B}\left(\mathcal{H}_{A}\right)$ such that $\widetilde{\phi}(x \otimes a)=\phi(x) a$ for all $x \in X$ and $a \in A$. By the Wittstock extension theorem and the automatic continuity in the $A, A$-topology $\widetilde{\phi}$ can be extended uniquely to a completely contractive $A$-bimodule map $\iota(\phi): X \stackrel{n p}{\otimes} A \rightarrow \mathrm{B}\left(\mathcal{H}_{A}\right)$. This defines an isometry $\iota: \operatorname{CB}\left(X, A^{\prime}\right) \rightarrow \mathrm{CB}_{A}\left(X \stackrel{n p}{\otimes} A, \mathrm{~B}\left(\mathcal{H}_{A}\right)\right)_{A}$. To see that $\iota$ is surjective, note that each $\mathrm{CB} A$-bimodule homomorphism $\psi: X \stackrel{n p}{\otimes} A \rightarrow \mathrm{B}\left(\mathcal{H}_{A}\right)$ necessarily maps $X \otimes 1$ into $A^{\prime}$, hence $\psi=\iota(\phi)$, where $\phi \in \mathrm{CB}\left(X, A^{\prime}\right)$ is defined by $\phi(x)=\psi(x \otimes 1)$. Finally, that $\iota$ is completely isometric follows from the identifications

$$
\mathrm{M}_{n}\left(\mathrm{CB}\left(X, A^{\prime}\right)\right)=\mathrm{CB}\left(X, \mathrm{M}_{n}\left(A^{\prime}\right)\right)
$$

and

$$
\begin{aligned}
\mathrm{M}_{n}\left(\mathrm{CB}_{A}\left(X \stackrel{n p}{\otimes} A, \mathrm{~B}\left(\mathcal{H}_{A}\right)\right)_{A}\right) & =\mathrm{CB}_{A}\left(X \stackrel{n p}{\otimes} A, \mathrm{M}_{n}\left(\mathrm{~B}\left(\mathcal{H}_{A}\right)\right)\right)_{A} \\
& =\mathrm{CB}_{A}\left(X \stackrel{n p}{\otimes} A, \mathrm{~B}\left(\mathcal{H}_{A}^{n}\right)\right)_{A}
\end{aligned}
$$

by replacing in the above argument $A$ with $A \otimes 1_{n}$ for each $n \in \mathbb{N}$. The rest of the proposition follows now from Theorem 5.1 .

When we deal with several operator spaces simultaneously, we denote the map $q: A \stackrel{e h}{\otimes} X \stackrel{e h}{\otimes} A \rightarrow X \stackrel{n p}{\otimes} A$ (defined in the Introduction) by $q_{X}$.

Corollary 5.3. Let $A$ a von Neumann algebra, $X^{\sharp}$ a dual operator space properly embedded in $\mathrm{B}(\mathcal{H})$ and denote by $\eta: \mathrm{T}(\mathcal{H}) \rightarrow X$ the map whose adjoint is the inclusion $X^{\sharp} \subseteq \mathrm{B}(\mathcal{H})$. Then the $\operatorname{map} q_{X}: A \stackrel{\text { eh }}{\otimes} X \stackrel{\text { eh }}{\otimes} A \rightarrow$ $X \stackrel{n p}{\otimes} A$ is (completely) quotient if and only if the map $\eta_{A}:=\eta \otimes 1: \mathrm{T}(\mathcal{H}) \stackrel{n p}{\otimes}$ $A \rightarrow X \stackrel{n p}{\otimes} A$ is (completely) quotient.

Proof. Consider the commutative diagram

$$
\begin{gathered}
A \stackrel{e h}{\otimes} \mathrm{T}(\mathcal{H}) \stackrel{e h}{\otimes} A \stackrel{q_{\mathrm{T}(\mathcal{H})}}{\longrightarrow} \mathrm{T}(\mathcal{H}) \stackrel{n p}{\otimes} A=\mathrm{NCB}_{A^{\prime}}\left(\mathrm{B}(\mathcal{H}) \bar{\otimes} A^{\prime}, \mathrm{B}\left(\mathcal{H}_{A}\right)\right)_{A^{\prime}} \\
\quad{ }_{\downarrow}{ }_{\eta_{A} \downarrow}^{\downarrow} \\
A \stackrel{e h}{\otimes} X \stackrel{e h}{\otimes} A \stackrel{q_{X}}{\longrightarrow} X \stackrel{n p}{\otimes} A=\mathrm{NCB}_{A^{\prime}}\left(X^{\sharp} \stackrel{F}{\otimes} A^{\prime}, \mathrm{B}\left(\mathcal{H}_{A}\right)\right)_{A^{\prime}}
\end{gathered}
$$

where $\sigma=1 \otimes \eta \otimes 1$ and the equalities follow from Proposition 5.2. From $[20,5.1(3), 4.2]$ we deduce that $q_{\mathrm{T}(\mathcal{H})}$ is a completely quotient map and the 
same holds for $\sigma$ by Proposition 4.2. It follows from the diagram that $q_{X}$ is a completely quotient map if and only if the same holds for $\eta_{A}$.

Corollary 5.4. Given an injective von Neumann algebra $A$, the map $q_{X}: A \stackrel{e h}{\otimes} X \stackrel{e h}{\otimes} A \rightarrow X \stackrel{n p}{\otimes} A=X \stackrel{A}{\otimes} A$ is a quotient map for all finitedimensional operator spaces $X$ if and only if there is a conditional expectation $E: \mathrm{B}\left(\mathcal{H}_{A}\right) \rightarrow A$ in the p.n. closure of the unit ball of $\operatorname{NCB}\left(\mathrm{B}\left(\mathcal{H}_{A}\right), A\right)$.

Proof. By the diagram in the proof of Corollary 5.3, $q_{X}$ is a quotient map if and only if the restriction map

$$
U:=\mathrm{NCB}_{A^{\prime}}\left(\mathrm{B}(\mathcal{H}) \bar{\otimes} A^{\prime}, \mathrm{B}\left(\mathcal{H}_{A}\right)\right)_{A^{\prime}} \rightarrow V:=\mathrm{NCB}_{A^{\prime}}\left(X^{\sharp} \stackrel{F}{\otimes} A^{\prime}, \mathrm{B}\left(\mathcal{H}_{A}\right)\right)_{A^{\prime}}
$$

is a quotient map. Note that $U=\operatorname{NCB}(\mathrm{B}(\mathcal{H}), A)$ (see $[20,4.2]$ ) and, if $X$ is finite-dimensional, the fact that injective von Neumann algebras are semidiscrete [4] implies by $[8,4.5]$ that $V=\mathrm{CB}\left(X^{\sharp}, A\right)$. Thus, $q_{X}$ is a quotient map for all finite-dimensional spaces $X$ if and only if the restriction $\operatorname{map} \operatorname{NCB}(\mathrm{B}(\mathcal{H}), A) \rightarrow \mathrm{CB}\left(X^{\sharp}, A\right)$ is quotient, which, by Proposition 4.3 and Corollary 4.5 , is the case if and only if there is a conditional expectation $E: \mathrm{B}\left(\mathcal{H}_{A}\right) \rightarrow A$ in the p.n. closure of the unit ball of $\operatorname{NCB}\left(\mathrm{B}\left(\mathcal{H}_{A}\right), A\right)$.

6. The case of algebras of type I. If $\mu$ is a positive countably additive measure on a ( $\sigma$-algebra on a) space $\Delta$ and $X$ is a Banach space we denote by $\mathcal{L}_{\infty}(\mu, X)$ the space of all essentially bounded (strongly) measurable functions from $\Delta$ to $X$ (see [6]). If $X$ is an operator space then $\mathcal{L}_{\infty}(\mu, X)$ is an operator space in a natural way. Given a countable set $\mathbb{J}$, we denote by $\widetilde{\mathcal{L}}_{\infty}\left(\mu, \mathrm{M}_{\mathbb{J}}(X)\right)$ the space of all functions $f=\left[f_{i j}\right]: \Delta \rightarrow \mathrm{M}_{\mathbb{J}}(X)$ such that the component functions $f_{i j}: \Delta \rightarrow X$ are measurable and $\|f\|$ is essentially bounded.

If $A$ is injective, then $X \stackrel{n p}{\otimes} A$ is just the closure $X \stackrel{A}{\otimes} A$ of $X \check{\otimes} A$ in the $A, A$-topology (since $C^{*}(X) \stackrel{\otimes}{\otimes} A=C^{*}(X) \check{\otimes} A$ by [8]), which by [23] is just the smallest strong $A, A$-bimodule containing $X \check{\otimes} A$.

LEMMA 6.1. Let $\mu$ be a positive finite measure on a space $\Delta, C=\mathcal{L}_{\infty}(\mu)$, $X$ an operator space, $\mathbb{J}$ a countable (or finite) set and $A=\mathrm{M}_{\mathbb{J}}(C)$. Then:

(i) $X \stackrel{C}{\otimes} C=\mathcal{L}_{\infty}(\mu, X)$.

(ii) $X \stackrel{A}{\otimes} A=\widetilde{\mathcal{L}}_{\infty}\left(\mu, \mathrm{M}_{\mathbb{J}}(X)\right)$.

Proof. (i) Put $Y=X \check{\otimes} C$ and represent the normal operator $C$-bimodule $Y$ as $Y \subseteq \mathrm{B}(\mathcal{H})$ for some Hilbert space $\mathcal{H}$ on which $C$ acts faithfully and normally. Since $C$ is abelian the closure $X \stackrel{C}{\otimes} C$ of $Y$ in the $C, C$-topology is the same as in the $\mathbb{C}, C$-topology (the two topologies have the same continuous functionals on the weak* closed submodule of $\mathrm{B}(\mathcal{H})$ consisting of 
elements commuting with $C$ ) and by $[22,2.2,5.3]$ this closure consists of all $f \in \mathrm{B}(\mathcal{H})$ such that there exists an orthogonal set $\left\{e_{i}: i \in \mathbb{I}\right\}$ of (non-zero) projections in $C$ with sum 1 such that $f e_{i} \in Y$. Since $\mu$ is finite, $\mathbb{I}$ must be countable. Regarding $Y$ as a subspace of $\mathcal{L}_{\infty}(\mu, X)$ in the usual way, we see that each $f e_{i}$ is in $\mathcal{L}_{\infty}(\mu, X)$, hence also $f=\sum_{i} f e_{i}$ is in $\mathcal{L}_{\infty}(\mu, X)$. Conversely, the Egorov theorem states that each $f \in \mathcal{L}_{\infty}(\mu, X)$ is a uniform limit of step functions outside a subset of arbitrary small measure and this implies that there is an orthogonal sequence $\left\{e_{i}\right\}$ of projections in $C$ such that $f e_{i} \in Y$ for all $i$, hence $f \in X \stackrel{C}{\otimes} C$.

(ii) We assume that $\mathbb{J}$ is infinite and identify $\mathbb{J}$ with $\mathbb{N}$. Let $p_{n} \in \mathrm{M}_{\mathbb{J}} \subseteq A$ be the projection onto the first $n$ coordinates, so that $\left(p_{n}\right)$ is a sequence of finite rank projections converging to 1 . Let $f=\left[f_{i j}\right] \in \widetilde{\mathcal{L}}_{\infty}\left(\mu, \mathrm{M}_{\mathbb{J}}(X)\right)$. By (i) the component functions $f_{i j}$ of $f$ are in $X \stackrel{C}{\otimes} C$, hence $p_{n} f p_{n} \in$ $\mathrm{M}_{n}(X \stackrel{C}{\otimes} C)$ for each $n$. Since $X \stackrel{A}{\otimes} A$ is a strong $A$-bimodule (hence a strong $C$-bimodule) containing $X \check{\otimes} A \supseteq \mathrm{M}_{n}(X \check{\otimes} C)$, it must contain $\mathrm{M}_{n}(X \stackrel{C}{\otimes} C)$, hence $p_{n} f p_{n} \in X \stackrel{A}{\otimes} A$ for all $n$. Since the sequence $\left(p_{n} f p_{n}\right)$ converges to $f$ in the $\mathrm{M}_{\mathbb{J}}, \mathrm{M}_{\mathbb{J}}$-topology (hence also in the $A, A$-topology), it follows that $f \in X \stackrel{A}{\otimes}$. This proves the inclusion $\widetilde{\mathcal{L}}_{\infty}\left(\mu, \mathrm{M}_{\mathbb{J}}(X)\right) \subseteq X \stackrel{A}{\otimes} A$ and the reverse inclusion follows from the easily verified fact that $\widetilde{\mathcal{L}}_{\infty}\left(\mu, \mathrm{M}_{\mathbb{J}}(X)\right)$ is a strong $A, A$-bimodule containing $X \check{\otimes} A$.

In the following theorem we have a restriction on the size of the operator space $X$; we do not know if it can be removed. In contrast, there is no restriction on the size of the von Neumann algebra $A$. To show this, we prove the theorem for a general $A$ of type I, although the proof is much shorter if $A$ has a separable predual (below, the separable case will be proved first).

TheOREM 6.2. If $A$ is a von Neumann algebra of type I and $X$ an operator space such that $X^{\sharp}$ can be properly embedded into $\mathrm{B}(\mathcal{H})$ with $\mathcal{H}$ separable, then $q: A \stackrel{e h}{\otimes} X \stackrel{e h}{\otimes} A \rightarrow X \stackrel{A}{\otimes} A$ is a completely quotient map.

Proof. Let $\iota: X^{\sharp} \rightarrow \mathrm{B}(\mathcal{H})$ be the inclusion, $\eta: \mathrm{T}(\mathcal{H}) \rightarrow X$ the completely quotient map with adjoint $\iota$ and set $T=\mathrm{T}(\mathcal{H})$. By Corollary 5.3 it suffices to prove that the map $\eta_{A}: T \stackrel{A}{\otimes} A \rightarrow X \stackrel{A}{\otimes} A$ is completely quotient. One can verify that if $A$ is a direct sum of von Neumann algebras $A_{i}$, then $Y \stackrel{A}{\otimes} A=\bigoplus_{i}\left(Y \stackrel{A_{i}}{\otimes} A_{i}\right)$ for each operator space $Y$. Thus, we may assume that $A=\mathrm{M}_{\mathbb{J}}(C)$ for some Abelian von Neumann algebra $C$ and some index set $\mathbb{J}$, since each von Neumann algebra of type $\mathrm{I}$ is a direct sum of algebras of such a form. We shall now divide the proof into three steps. 
1. Suppose that $A$ has a separable predual. Then $\mathbb{J}$ is countable (hence we will assume that $\mathbb{J}=\mathbb{N}$ ) and (up to isomorphism) $C=\mathcal{L}_{\infty}(\mu)$ for some finite complete Borel measure on a compact metric space $\Delta$. By Lemma 6.1, $X \stackrel{A}{\otimes} A=\widetilde{\mathcal{L}}_{\infty}\left(\mu, \mathrm{M}_{\mathbb{J}}(X)\right)$. Let $f \in \widetilde{\mathcal{L}}_{\infty}\left(\mu, \mathrm{M}_{\mathbb{J}}(X)\right)$ and $\varepsilon \in(0,1)$. Put $B=\mathrm{M}_{\mathbb{J}}$ and let $\eta_{\mathbb{J}}: \mathrm{M}_{\mathbb{J}}(T) \rightarrow \mathrm{M}_{\mathbb{J}}(X)$ be the map induced by $\eta$. Since $\mathrm{M}_{\mathbb{J}}(X)=$ $\operatorname{NCB}\left(X^{\sharp}, \mathrm{M}_{\mathbb{J}}\right)$ and $\mathrm{M}_{\mathbb{J}}(T)=\operatorname{NCB}\left(\mathrm{B}(\mathcal{H}), \mathrm{M}_{\mathbb{J}}\right)$ and $X^{\sharp}$ is properly embedded in $\mathrm{B}(\mathcal{H}), \eta_{\mathbb{J}}$ is a quotient map. Therefore for each $\lambda \in \Delta$ there exists an element $t(\lambda) \in \mathrm{M}_{\mathbb{J}}(T)$ such that $\eta_{\mathbb{J}}(t(\lambda))=f(\lambda)$ and $\|t(\lambda)\|<\|f(\lambda)\|+\varepsilon$. To show that $t(\lambda)$ can be chosen in a measurable way, we may assume (by changing it on a set of measure 0 , see [16, p. 1032]) that $f$ is a Borel map. Let $V$ be the closed ball in $\mathrm{M}_{\mathbb{J}}(T)$ with centre 0 and radius $\|f\|+\varepsilon$, equipped with the topology determined by the family of seminorms $t \mapsto\left\|p_{n} t p_{n}\right\|$, where $p_{n} \in \mathrm{M}_{\mathbb{J}}$ is the projection onto the first $n$ coordinates for each $n \in \mathbb{J}=\mathbb{N}$. (It is not difficult to verify that this is just the $B, B$-topology on $V$.) Then $V$ is a complete separable metrizable space. Since the map $\eta_{\mathbb{J}}: \mathrm{M}_{\mathbb{J}}(T) \rightarrow \mathrm{M}_{\mathbb{J}}(X)$ is continuous where both spaces carry the $B, B$-topology, the set

$$
S=\left\{(\lambda, t) \in \Delta \times V: f(\lambda)=\eta_{\mathbb{J}}(t)\right\}
$$

is a Borel subset of $\Delta \times V$. By the principle of measurable selection (see [16, p. 1041]) there exists a measurable map $g: \Delta \rightarrow V$ such that $\eta_{\mathbb{J}}(g(\lambda))=f(\lambda)$ for all $\lambda \in \Delta$. The topology in $V$ is such that measurability of $g$ means that the components $g_{i j}$ of $g$ are measurable functions from $\Delta$ into $X$, hence $g \in \widetilde{\mathcal{L}}_{\infty}\left(\mu, \mathrm{M}_{\mathbb{J}}(T)\right)=T \stackrel{A}{\otimes} A$. The identity $\eta_{\mathbb{J}}(g(\lambda))=f(\lambda)$ a.e. means that $\eta_{A}(g)=f$ and, since $\|g\| \leq\|f\|+\varepsilon$, this proves that $\eta_{A}$ is a quotient map. Thus $q$ is a quotient map. Replacing in the above argument $A$ with $\mathrm{M}_{n}(A)$ for all $n \in \mathbb{N}$, we see that $q$ is in fact a completely quotient map. This proves the theorem in the case where $A$ has a separable predual.

2. Suppose now that $A$ is countably decomposable (but not necessarily with a separable predual). Since bounded subsets of $X \stackrel{A}{\otimes} A$ are metrizable in the $A, A$-topology and each $w \in X \stackrel{A}{\otimes} A$ is in the $A, A$-closure of some bounded subset of $X \check{\otimes} A$ by [23], each $w \in X \stackrel{A}{\otimes} A$ is in fact a limit in the $A, A$-topology of a sequence of elements $w_{n} \in X \check{\otimes} A$. We claim that there exists a countably generated type I von Neumann subalgebra $A_{0}$ of $A$ such that $w \in X \stackrel{A_{0}}{\otimes} A_{0}$. To see this, let $\omega$ be a faithful normal state on $A$. Since $w_{n}$ is a Cauchy sequence in the $A, A$-topology, it follows from $[23,3.1]$ that we have $w_{n}-w_{m}=a_{m, n} y_{m, n}+z_{m, n} b_{m, n}$ for some bounded sequences $\left(y_{m, n}\right),\left(z_{m, n}\right) \subseteq X \check{\otimes} A$ and $\left(a_{m, n}\right),\left(b_{m, n}\right) \subseteq A^{+}$ such that $\omega\left(a_{m, n}^{2}\right)$ and $\omega\left(b_{m, n}^{2}\right)$ tend to 0 as $m$ and $n$ tend to $\infty$. Let $A_{0}$ be a countably generated von Neumann subalgebra of $A$ such that all 
$w_{n}, y_{m, n}$ and $z_{m, n}$ are in $X \check{\otimes} A_{0}$ and all $a_{m, n}$ and $b_{m, n}$ are in $A_{0}$. Further, by replacing $A_{0}$ with the smallest von Neumann subalgebra of $A$ which contains $A_{0}$ and is invariant for the modular group corresponding to the state $\omega$, we know by [30] that there is a faithful normal conditional expectation from $A$ to $A_{0}$, hence $A_{0}$ is of type I by [31]. By construction, $\left(w_{n}\right)$ is a Cauchy sequence in the $A_{0}, A_{0}$-topology of $X \stackrel{A_{0}}{\otimes} A_{0}$, hence (since a closed ball of $X \stackrel{A_{0}}{\otimes} A_{0}$ is a complete metric space in the $A_{0}, A_{0}$-topology by $[22,5.5]$ and $\left.[23,3.1]\right)$ the sequence has a limit $\widetilde{w}$. But $\widetilde{w}$ is then also the limit of the sequence $\left(w_{n}\right)$ in the (weaker) $A, A$-topology of $X \stackrel{A}{\otimes} A$, hence $\widetilde{w}=w$. This proves the claim. Since a $\sigma$-finite countably generated von Neumann algebra has a separable predual, by what we have already proved there is an element $t \in T \stackrel{A_{0}}{\otimes} A_{0} \subseteq T \stackrel{A}{\otimes} A$ with $\|t\| \leq\|w\|+\varepsilon$ and $\eta_{A}(t)=w$. This proves that $\eta_{A}$ is a quotient map if $A$ is $\sigma$-finite.

3. In general, if $A$ is not countably decomposable, we can reduce the problem to the $\sigma$-finite case as follows. First we may regard $X \check{\otimes} A$ (and consequently $X \stackrel{A}{\otimes} A$ ) as a subbimodule in $\operatorname{NCB}\left(X^{\sharp}, A\right)$ in the well known canonical way. For each $w \in \operatorname{NCB}\left(X^{\sharp}, A\right)$ the von Neumann subalgebra $A_{w}$ of $A$ generated by the range of $w$ contains a countable weak ${ }^{*}$ dense subset (since $X^{\sharp}$ does), therefore for each vector $\xi \in \mathcal{H}_{A}$ the projection $p_{\xi}$ with range $\left[A^{\prime} A_{w} \xi\right]$ is $\sigma$-finite in $A$ and clearly $p_{\xi} \in A \cap A_{w}^{\prime}$. This implies, by a standard maximality argument, that $w$ can be decomposed as a sum $w=\sum_{i} p_{i} w$ for some orthogonal family $\left(p_{i}\right)$ of $\sigma$-finite (in $A$ ) projections $p_{i} \in A \cap A_{w}^{\prime}$ and each $p_{i} w$ can be regarded as an element of $\operatorname{NCB}\left(X^{\sharp}, A_{i}\right)$, where $A_{i}=p_{i} A p_{i}$. Further, if $w \in X \stackrel{A}{\otimes} A$, then $p_{i} w \in X \stackrel{A_{i}}{\otimes} A_{i}$ for all $i$, hence, by what we have already proved, there exist $t_{i} \in T \stackrel{A_{i}}{\otimes} A_{i}$ such that $\eta_{A_{i}}\left(t_{i}\right)=p_{i} w$ and $\left\|t_{i}\right\|<\left\|p_{i} w\right\|+\varepsilon$. Then $t:=\sum t_{i} \in \bigoplus_{i}\left(T \stackrel{A_{i}}{\otimes} A_{i}\right) \subseteq T \stackrel{A}{\otimes} A$ is such that $\eta_{A}(t)=w$ and $\|t\|<\|w\|+\varepsilon$. This proves that $\eta_{A}$ is a quotient map; that it is in fact completely quotient follows again by replacing in this argument $A$ with $\mathrm{M}_{n}(A)$ for all $n \in \mathbb{N}$.

We do not know if in Theorem 6.2 , the restriction that $X$ can be properly embedded into $\mathrm{B}(\mathcal{H})$ for a separable $\mathcal{H}$ can be removed. This condition is satisfied in particular for all finite-dimensional operator spaces (Corollary 4.4) and all operator spaces $X$ such that $X^{\sharp}$ can be realised as a subspace in some $\mathrm{B}(\mathcal{H})$ for a separable $\mathcal{H}$ so that $X^{\sharp} \cap \mathrm{K}(\mathcal{H})$ is weak ${ }^{*}$ dense in $X^{\sharp}$.

REMARK 6.3. If $A$ is a finite von Neumann algebra of type I, then the map $q: A \stackrel{e h}{\otimes} X \stackrel{e h}{\otimes} A \rightarrow X \stackrel{n p}{\otimes} A$ is completely quotient for each operator 
space $X$. One way to show this (indirectly suggested to me by Christian Le Merdy) is to observe that if in the first part of the proof of Theorem $6.2, \mathbb{J}$ is finite then $\widetilde{\mathcal{L}}_{\infty}\left(\mu, \mathrm{M}_{\mathbb{J}}(X)\right)=\mathcal{L}_{\infty}\left(\mu, \mathrm{M}_{\mathbb{J}}(X)\right)$, where $\mu$ is a finite countably additive positive measure on a space $\Delta$. Thus each $f \in$ $\widetilde{\mathcal{L}}_{\infty}\left(\mu, \mathrm{M}_{\mathbb{J}}(X)\right)$ can be approximated uniformly by functions of the form $\sum_{j=1}^{\infty} \chi_{\Delta_{j}} x_{j}$, where $x_{j} \in \mathrm{M}_{\mathbb{J}}(X)$ and $\chi_{\Delta_{j}}$ are the characteristic functions of disjoint measurable subsets $\Delta_{j}$ of $\Delta$ (this follows from the proof of the Pettis measurability theorem, $[6$, p. 42]), hence (by the proof of the open mapping theorem) $f$ can be lifted in an appropriate way to an element of $\widetilde{\mathcal{L}}_{\infty}\left(\mu, \mathrm{M}_{\mathbb{J}}(T)\right)$.

From Theorem 6.2, Corollary 5.4, Corollary 4.5 and Proposition 4.3 we deduce the following consequence.

Corollary 6.4. For each von Neumann algebra A of type I and a finitedimensional subspace $X^{\sharp} \subseteq \mathrm{B}(\mathcal{H})$ every $C B$ map $\phi: X^{\sharp} \rightarrow A$ with $\|\phi\|_{\mathrm{cb}}<1$ can be extended to a normal map $\psi: \mathrm{B}(\mathcal{H}) \rightarrow A$ with $\|\psi\|_{\mathrm{cb}}<1$.

Theorem 6.2 (together with Corollary 5.3) implies that if $X$ is a finitedimensional operator space and $q: \mathrm{T}(\mathcal{H}) \rightarrow X$ is a completely quotient map then the induced map $\mathrm{T}(\mathcal{H}) \stackrel{A}{\otimes} A \rightarrow X \check{\otimes} A$ is also completely quotient if $A$ is of type I. If we replace $\mathrm{T}(\mathcal{H}) \stackrel{A}{\otimes} A$ with the usual spatial tensor product $\mathrm{T}(\mathcal{H}) \otimes A$ the result is no longer true for all finite-dimensional operator spaces $X$ even in the case $A=\mathrm{B}(\mathcal{H})$. After we had already found a direct proof of the following proposition, $\mathrm{N}$. Ozawa kindly informed us that the first part of it can also be deduced from his results in [25]. We give below our original proof. We refer to [27] for the definition of 1-exact operator spaces that are used in the following proposition.

Proposition 6.5. Let $\eta: \mathrm{T}(\mathcal{H}) \rightarrow X$ be a completely quotient map, where $X$ is finite-dimensional. Then the induced map $\eta_{1}:=\eta \otimes 1: \mathrm{T}(\mathcal{H}) \ddot{\otimes}$ $\mathrm{B}(\mathcal{H}) \rightarrow X \check{\otimes} \mathrm{B}(\mathcal{H})$ is completely quotient if and only if the dual $X^{\sharp}$ of $X$ is 1-exact. In this case the map $\eta \otimes 1: \mathrm{T}(\mathcal{H}) \check{\otimes} A \rightarrow X \check{\otimes} A$ is completely quotient for each injective operator space $A$.

Proof. Put $T=\mathrm{T}(\mathcal{H}), B=\mathrm{B}(\mathcal{H})$ and identify $B$ with $\mathrm{M}_{\mathbb{J}}$ for some index set $\mathbb{J}$. Note that taking adjoints induces a complete isometry between $\mathrm{CB}(T, Y)$ and $\operatorname{NCB}\left(Y^{\sharp}, B\right)=\mathrm{M}_{\mathbb{J}}(Y)$ for each operator space $Y$; under this identification the subspace $Y \check{\otimes} B$ of $\mathrm{M}_{\mathbb{J}}(Y)$ corresponds to the closure $\overline{\operatorname{FCB}(T, Y)}^{\mathrm{cb}}$ of finite rank operators $\operatorname{FCB}(T, Y)$.

For a finite-dimensional $X$, if the map $\eta_{1}: T \check{\otimes} B \rightarrow X \check{\otimes} B$ is quotient, then by the identification of the previous paragraph this means that the corestriction map $r: \overline{\operatorname{FCB}(T, T)}{ }^{\mathrm{cb}} \rightarrow \operatorname{FCB}(T, X), r(\phi)=\eta \phi$, is a quotient 
map. In particular, given $\varepsilon>0$, there exists $\phi \in \overline{\mathrm{FCB}(T)}^{\mathrm{cb}}$ such that $\eta \phi=\eta$ and $\|\phi\|_{\mathrm{cb}}<1+\varepsilon / 8$. Choose $\psi \in \operatorname{FCB}(T)$ with $\|\phi-\psi\|_{\mathrm{cb}}<\varepsilon / 8$, hence $\|\psi\|_{\mathrm{cb}}<1+\varepsilon / 4$. Put $S=\operatorname{im} \psi$, a finite-dimensional subspace of $T$.

Given a map $\theta: X \rightarrow Y$ between operator spaces we shall denote for each $n$ the induced map $\theta \otimes 1: \mathrm{M}_{n}(X) \rightarrow \mathrm{M}_{n}(Y)$ by $\theta$ again for simplicity of notation.

Observe that for each $n \in \mathbb{N}$ and each $t$ in the unit ball of $\mathrm{M}_{n}(T)$ there exists an $s$ in the unit ball of $\mathrm{M}_{n}(S)$ such that $\|\phi(t)-s\|<\varepsilon / 2$. (Indeed, since $\|\phi(t)-\psi(t)\|<\varepsilon / 4$ and $\|\psi(t)\|<1+4^{-1} \varepsilon$, we can choose $s=$ $\left.\left(1+4^{-1} \varepsilon\right)^{-1} \psi(t).\right)$

Since $\eta$ is completely quotient, given $n$ and $x \in \mathrm{M}_{n}(X)$ with $\|x\|=1$, there exists $t \in \mathrm{M}_{n}(T)$ such that $\eta(t)=x$ and $\|t\|<1+2^{-1} \varepsilon$. Thus $t_{1}:=(1+$ $\left.2^{-1} \varepsilon\right)^{-1} t$ satisfies $\left\|t_{1}\right\|<1$ and $\left\|x-\eta\left(t_{1}\right)\right\|<\varepsilon / 2$. By the previous paragraph there exists an $s_{1} \in \mathrm{M}_{n}(S)$ such that $\left\|s_{1}\right\| \leq 1$ and $\left\|\phi\left(t_{1}\right)-s_{1}\right\|<\varepsilon / 2$. Thus,

$$
\left\|x-\eta\left(s_{1}\right)\right\|=\left\|x-\eta\left(t_{1}\right)+\eta\left(\phi\left(t_{1}\right)-s_{1}\right)\right\|<\varepsilon .
$$

This means that for each $y \in \mathrm{M}_{n}(X)$ there exists an element $s \in \mathrm{M}_{n}(S)$ such that $\|s\| \leq\|y\|$ and $\|y-\eta(s)\| \leq \varepsilon\|y\|$. Applying this successively to elements $x, x-\eta\left(s_{1}\right), \ldots$, we find a sequence of elements $s_{k} \in \mathrm{M}_{n}(S)$ such that $\left\|s_{k}\right\| \leq \varepsilon^{k-1}$ and $\left\|x-\eta\left(s_{1}\right)-\ldots-\eta\left(s_{k}\right)\right\| \leq \varepsilon^{k}$. For $s:=\sum s_{k}$ we now have $\eta(s)=x$ and $\|s\| \leq(1-\varepsilon)^{-1}$.

So we have proved that for each $\varepsilon>0$ there exists a finite-dimensional subspace $S$ of $T$ such that for each $n$ and $x \in \mathrm{M}_{n}(X)$ there is an element $s \in \mathrm{M}_{n}(S)$ with $\eta(s)=x$ and $\|s\| \leq(1-\varepsilon)^{-1}\|x\|$. We can replace $S$ in this statement by $T_{m}$, the predual of $\mathrm{M}_{m}$, for some $m \in \mathbb{N}$; this follows from the fact that for each finite-dimensional subspace $S$ of $T$ there exists $m \in \mathbb{N}$ such that $S$ is $\varepsilon$-almost completely isometric to a subspace of $T_{m}$ (see [11] for a more general result of this sort). By duality this implies that the completely bounded Banach-Mazur distance from $X^{\sharp}$ to some subspace of $\mathrm{M}_{m}$ is dominated by $(1-\varepsilon)^{-2}$ (see the proof of Theorem 4.1.8 in [12]), hence $X^{\sharp}$ is 1 -exact since $\varepsilon>0$ was arbitrary.

Conversely, if $X^{\sharp}$ is 1-exact (that is, arbitrarily close in the CB BanachMazur distance to a subspace of $\mathrm{M}_{m}$ for some $m$ ), then by duality, given $\varepsilon>0$, there exists an $m$ and a complete contraction $\tau: T_{m} \rightarrow X$ such that the induced map $T_{m} / \operatorname{ker} \tau \rightarrow X$ is invertible with the CB norm of the inverse less than $1+\varepsilon$. Since $T_{m}$ is a projective operator space [2], there exists a linear map $\phi: T_{m} \rightarrow T$ with $\eta \phi=\tau$ and $\|\phi\|_{\mathrm{cb}}<1+\varepsilon$. Tensoring with $A$, it now suffices to show that the map $\tau \otimes 1: T_{m} \check{\otimes} A \rightarrow X \check{\otimes} A$ is $\varepsilon$-almost completely quotient, and this translates to the fact that the map $r: \mathrm{CB}\left(\mathrm{M}_{m}, A\right) \rightarrow \mathrm{CB}\left(X^{\sharp}, A\right), r(\theta)=\theta \tau^{\sharp}$ (where $\tau^{\sharp}$ is the adjoint of $\tau$ ), is almost completely quotient, which is a consequence of the extension theorem for CB maps. 
Corollary 6.6. If $X$ is a finite-dimensional operator space whose dual $X^{\sharp}$ is 1-exact, then the map $q: A \stackrel{\text { eh }}{\otimes} \underset{\text { eh }}{\otimes} A \rightarrow X \check{\otimes} A$ is completely quotient for each injective von Neumann algebra $A$.

Proof. Since $\mathrm{T}(\mathcal{H}) \check{\otimes} A \subseteq \mathrm{T}(\mathcal{H}) \stackrel{n p}{\otimes} A$ and $X \check{\otimes} A=X \stackrel{n p}{\otimes} A$, the map $\eta_{A}: \mathrm{T}(\mathcal{H}) \stackrel{n p}{\otimes} A \rightarrow X \stackrel{n p}{\otimes} A$ is completely quotient by Proposition 6.5 , hence the result follows from Corollary 5.3.

\section{References}

[1] W. B. Arveson, Notes on extensions of $C^{*}$-algebras, Duke Math. J. 44 (1977), 329-355.

[2] D. P. Blecher, The standard dual of an operator space, Pacific J. Math. 153 (1992), $15-30$.

[3] D. P. Blecher and R. R. Smith, The dual of the Haagerup tensor product, J. London Math. Soc. 45 (1992), 126-144.

[4] A. Connes, On the equivalence between injectivity and semidiscreteness for operator algebras, in: Algèbres d'Opérateurs et leurs Applications en Physique Mathématique (Marseille, 1977), in: Colloq. Internat. CNRS 274, 1979, 107-112.

[5] J. B. Conway, A Course in Operator Theory, Grad. Stud. Math. 21, Amer. Math. Soc., Providence, RI, 2000.

[6] J. Diestel and J. J. Uhl, Vector Measures, Math. Surveys 15, Amer. Math. Soc., Providence, RI, 1977.

[7] E. G. Effros and A. Kishimoto, Module maps and Hochschild-Johnson cohomology, Indiana Univ. Math. J. 36 (1987), 257-276.

[8] E. G. Effros and E. C. Lance, Tensor products of operator algebras, Adv. Math. 25 (1977), 1-34.

[9] E. G. Effros and Z.-J. Ruan, Representation of operator bimodules and their applications, J. Operator Theory 19 (1988), 137-157.

[10] - - -, Operator convolution algebras: An approach to quantum groups, preprint.

[11] - -, $\mathcal{O} \mathcal{L}_{p}$ spaces, in: Operator Algebras and Operator Theory, Contemp. Math. 228, Amer. Math. Soc., 1998, 51-77.

[12] -, 一, Operator Spaces, London Math. Soc. Monographs 23, Oxford Univ. Press, Oxford, 2000.

[13] U. Haagerup, The standard form of von Neumann algebras, Math. Scand. 37 (1975), $271-283$.

[14] H. Hofmeier and G. Wittstock, A bicommutant theorem for completely bounded module homomorphisms, Math. Ann. 308 (1997), 141-154.

[15] M. Junge and C. Le Merdy, Factorization through matrix spaces for finite rank operators between $C^{*}$-algebras, Duke Math. J. 100 (1999), 299-319.

[16] R. V. Kadison and J. R. Ringrose, Fundamentals of the Theory of Operator Algebras, Vols. 1, 2, Academic Press, London, 1983, 1986.

[17] G. G. Kasparov, Hilbert $C^{*}$-modules: theorems of Stinespring and Voiculescu, J. Operator Theory 4 (1980), 133-150.

[18] E. Kirchberg and N. C. Phillips, Embedding of exact $C^{*}$-algebras in the Cuntz algebra $\mathcal{O}_{2}$, J. Reine Angew. Math. 525 (2000), 17-53. 
[19] C. Le Merdy, Finite rank approximation and semidiscreteness for linear operators, Ann. Inst. Fourier (Grenoble) 49 (1999), 1869-1901.

[20] C. Le Merdy and B. Magajna, A factorization problem for normal completely bounded mappings, J. Funct. Anal. 181 (2001), 313-345.

[21] B. Magajna, Strong operator modules and the Haagerup tensor product, Proc. London Math. Soc 74 (1997), 201-240.

[22] B. Magajna, A topology for operator modules over $W^{*}$-algebras, J. Funct. Anal. 154 (1998), 17-41.

[23] - $C^{*}$-convex sets and completely bounded bimodule homomorphisms, Proc. Roy. Soc. Edinburgh Sect. A 130 (2000), 375-387.

[24] Q. Na, Standard duals of operator bimodules, J. Funct. Anal. 135 (1996), 132156.

[25] N. Ozawa, On the lifting property for universal $C^{*}$-algebras of operator spaces, preprint, 2000.

[26] V. I. Paulsen, Completely Bounded Maps and Dilations, Pitman Res. Notes Math. Ser. 146, Longman, 1986.

[27] G. Pisier, Exact operator spaces, Astérisque 232 (1995), 159-186.

[28] - , Introduction to the Theory of Operator Spaces, a book to appear, Cambridge Univ. Press.

[29] C. Pop, Bimodules normés représentables sur des espaces hilbertiens, Ph.D. thesis, Univ. d'Orléans, 1999.

[30] M. Takesaki, Conditional expectations in von Neumann algebras, J. Funct. Anal. 9 (1972), 306-321.

[31] J. Tomiyama, On the projections of norm one in $W^{*}$-algebras, III, Tohoku Math. J. 11 (1959), 125-129.

[32] D. V. Voiculescu, A non-commutative Weyl-von Neumann theorem, Rev. Roumaine Math. Pures Appl. 21 (1976), 97-113.

[33] G. Wittstock, Ein operatorwertiger Hahn-Banach Satz, J. Funct. Anal. 40 (1981), $127-150$.

Department of Mathematics

University of Ljubljana

Jadranska 19

Ljubljana 1000, Slovenia

E-mail: Bojan.Magajna@fmf.uni-lj.si

Received June 21, 2001

Revised version June 3, 2002 